\title{
Instanton Moduli in String Theory
}

\author{
Evgeny I. Buchbinder, ${ }^{1}$ Burt A. Ovrut ${ }^{2}$ and Rene Reinbacher ${ }^{3}$ \\ ${ }^{1}$ School of Natural Sciences, Institute for Advanced Study \\ Einstein Drive, Princeton, NJ 08540, USA \\ ${ }^{2}$ Department of Physics and Astronomy, University of Pennsylvania \\ Philadelphia, PA 19104, USA \\ ${ }^{3}$ Department of Mathematics, Harvard University, Cambridge, MA 02138, USA
}

\begin{abstract}
Expressions for the number of moduli of arbitrary $S U(n)$ vector bundles constructed via Fourier-Mukai transforms of spectral data over Calabi- Yau threefolds are derived and presented. This is done within the context of simply connected, elliptic Calabi-Yau threefolds with base $\mathbb{F}_{r}$, but the methods have wider applicability. The condition for a vector bundle to possess the minimal number of moduli for fixed $r$ and $n$ is discussed and an explicit formula for the minimal number of moduli is presented. In addition, transition moduli for small instanton phase transitions involving non-positive spectral covers are defined, enumerated and given a geometrical interpretation.
\end{abstract}




\section{Introduction}

It was shown in [1, 2, 3, 4, 5] that compactifying Hořava-Witten theory [6, 7] on a Calabi-Yau threefold, $X$, with an appropriate holomorphic vector bundle, $V$, can lead, on the observable brane, to realistic GUT and standard-like models at low energy. It is clear that in this context instantons with the "standard embedding", that is, vector bundles where $V=T X$, do not play a special role and that one should consider more generalized vector bundles. Based on the work in [8, 9], large classes of such bundles were constructed. In [10, 11], stable holomorphic vector bundles with structure group $S U(n)$ over simply connected elliptic Calabi-Yau threefolds were presented. These lead to GUT models in four-dimensions with gauge groups $S U(5)$ and $\operatorname{Spin}(10)$, for example. In a series of papers [12, 13, 14, 15, 16, 17, 18, stable holomorphic vector bundles with structure group $S U(n)$ were constructed, using new methods, over Calabi-Yau threefolds with non-trivial fundamental group. By combining these bundles with Wilson lines, one can produce quasi-realistic low energy models containing the standard model gauge group $S U(3)_{C} \times S U(2)_{L} \times U(1)_{Y}$. Various properties of these generalized heterotic vacua have been studied, including the moduli space and BPS spectrum [19, 20, 21, 22, 23] of the associated five-branes, small instanton phase transitions [24, 25, 26, 27, 28, 29], non-perturbative superpotentials [30, 31, 32, 33, 34, 35, 36, 37, supersymmetry breaking [38, 39, 40, 41, 42, 43] and moduli stabilization [4, 45, 46, 47, 48. Recently, it was shown how to compute the particle spectrum produced by generalized vector bundles, both in GUT theories [49, 50] and in theories of the standard model [51]. Such theories also impact early universe cosmology. In particular, they underlie the formulation of Ekpyrotic cosmology [52, 53, 54].

An important aspect of generalized vector bundles is their moduli space. Vector bundle moduli appear as uncharged complex scalar fields in the low energy effective theory and, as such, strongly impact particle phenomenology and cosmology. The computation of the number and properties of these moduli is a non-trivial exercise in sheaf cohomology. This was carried out for generalized bundles on simply connected elliptic Calabi-Yau threefolds in [27], under the simplifying assumption that the spectral covers associated with these bundles are "positive". Within this context, the moduli associated with phase transitions, "transition" moduli, were defined and studied [27]. Furthermore, in [35, 36] the contribution of vector bundle moduli to non-perturbative superpotentials was explicitly computed. Unfortunately, the number of positive spectral covers is only a small subset of all allowed covers. In addition, one can show that most phenomenologically acceptable heterotic vacua correspond to 
spectral covers that are explicitly not positive. It is important, therefore, to generalize the results of [27] so as to compute the number and properties of the moduli of vector bundles associated with non-positive spectral covers. This will be accomplished in this paper, along with a description of the transition moduli.

Specifically, we do the following. In Section 2, the construction of stable holomorphic vector bundles with structure group $S U(n)$ on simply connected elliptic Calabi-Yau threefolds is reviewed. For concreteness, we will always work within the context of Calabi-Yau threefolds with base surface $\mathbb{F}_{r}$ and $r=0,1,2$. The expression for the number of quark/lepton generations is presented and the condition for anomaly cancellation, including the structure of the associated five-branes, is discussed. We close the section by giving two explicit examples of anomaly free, $S U(5)$ GUT theories with three families of quarks and leptons.

Instanton moduli for vector bundles constructed from spectral data via a Fourier-Mukai transform are discussed in Section 3. We begin by giving the definition of these moduli and a generic formula for enumerating them. The notion of positive spectral cover is then reviewed and the expression for the number of vector bundle moduli in this context, originally computed in [27], is presented. In the next subsection, we generalize these results to arbitrary spectral covers, specifically to the large set of covers that are non-positive. Expressions for the number of vector bundle moduli for arbitrary spectral covers are comprehensively derived. It is shown that, unlike the positive case, the spectral line bundles may contribute moduli for some non-positive covers. We then re-examine the two three family GUT models presented earlier, show that they have non-positive spectral covers and compute the number of vector bundle moduli in each case. The condition under which a vector bundle has the minimal number of moduli for fixed parameters $r$ and $n$ is discussed. Such a bundle is always non-positive. An explicit formula for the minimal number of moduli is presented. We close the section by giving an example of a GUT theory with a minimal number of moduli, and compare it to the two previous examples.

Section 4 is devoted to the study of transition moduli. We review the definition and enumeration of transition moduli in small instanton transitions involving vector bundles with positive spectral covers. These notions are then extended to the case of non-positive spectral covers and a geometric interpretation of the associated transition moduli is given, including the role of spectral line bundle moduli. Several explicit examples illustrating all of these notions are then presented. Concluding remarks are made in Section 5. In addition, we give important consistency checks of our methods in Appendix A and useful linear algebra relations are presented in Appendix B. 
An interesting result of our calculations is that vector bundles with non-positive spectral covers have far fewer moduli than bundles with positive cover, typically by a factor of about a half. Though very promising in reducing the moduli abundance problem, the number is still rather large. However, when our methods are applied to vector bundles over non-simply connected Calabi-Yau threefolds, we expect this number to be dramatically reduced. This will be discussed elsewhere.

\section{Heterotic String on Elliptically Fibered Calabi-Yau Threefolds}

\subsection{Vector Bundles on Elliptically Fibered Calabi-Yau Threefolds}

We consider strongly coupled heterotic string theory [6, 17, 1] compactified on Calabi-Yau threefolds, $X$, structured as elliptic curves fibered over a base, B. The maps $\pi: X \rightarrow B$ and $\sigma: B \rightarrow X$ are the natural projection and zero section respectively. All such Calabi-Yau threefolds are simply connected. The requirement that $X$ be a Calabi-Yau space constrains the first Chern class of the tangent bundle to vanish,

$$
c_{1}(X)=0
$$

This restricts the base space $B$ to be either del Pezzo, Hirzebruch, Enrique or certain blowups of a Hirzebruch surface [55]. The second Chern class of the tangent bundle of $X$ can be shown to be 8 ]

$$
c_{2}(X)=\pi^{*}\left(c_{2}(B)+11 c_{1}(B)^{2}\right)+12 \sigma \cdot \pi^{*} c_{1}(B),
$$

where $c_{1}(B)$ and $c_{2}(B)$ are the first and second Chern classes of the tangent bundle of $B$.

To specify a heterotic string vacuum, one has to present, in addition to the threefold $X$, a holomorphic instanton, A, satisfying the hermitian Yang-Mills equations,

$$
\begin{aligned}
& F_{a b}=F_{\bar{a} \bar{b}}=0, \\
& g^{a \bar{b}} F_{a \bar{b}}=0 .
\end{aligned}
$$

If the instanton is indexed in the Lie Algebra of group $G$, then the gauge group of the effective four-dimensional theory will be the commutant of $G$ in $E_{8}$. A theorem of Donaldson and Uhlenbeck-Yau [56, 57] states that the existence of such an instanton is equivalent to constructing a stable, holomorphic vector bundle, $V$, on $X$, in the sense that such a bundle 
admits a connection satisfying 2.3. This theorem also asserts that all possible deformations of the Yang-Mills instanton are in one-to-one correspondence with moduli of the stable, holomorphic vector bundle. Since no hermitian Yang-Mills solutions on Calabi-Yau threefolds have been explicitly constructed, the vector bundle approach seems to be the only practical way to study the physics of instantons and their moduli in heterotic compactifications. A general construction of vector bundles on elliptically fibered Calabi-Yau manifolds was given in [8, 9. In this paper, we consider stable, holomorphic vector bundles with structure group $S U(n)$. Such bundles over an elliptically fibered Calabi-Yau manifold can be explicitly constructed from two mathematical objects, a divisor $\mathcal{C}$ of $X$, called the spectral cover, and a line bundle $\mathcal{N}$ on $\mathcal{C}$.

A spectral cover is a surface in $X$ that is an $n$-fold cover of the base $B$. The general form is

$$
\mathcal{C} \in\left|n \sigma+\pi^{*} \eta\right|
$$

where $\left|n \sigma+\pi^{*} \eta\right|$ denotes the linear system of $\mathcal{O}_{X}\left(n \sigma+\pi^{*} \eta\right)$ and $\eta$ is a curve class in the base. For this vector bundle to be stable, we require that the spectral cover be irreducible. The linear system $\left|n \sigma+\pi^{*} \eta\right|$ will contain such irreducible surfaces if the following conditions are met [8, 26].

- $\pi^{*} \eta$ is an irreducible divisor in $X$. This condition can be satisfied if one demands that the linear system $|\eta|$ be base-point free in $B$.

- $c_{1}\left(\pi^{*} K_{B}^{\otimes n} \otimes \mathcal{O}_{X}\left(\pi^{*} \eta\right)\right)$ is effective in $H_{4}(X, \mathbb{Z})$. This condition is equivalent of saying that

$$
\eta-n c_{1}(B)
$$

is effective in $B$.

To make these concepts more concrete, we take the base to be the Hirzebruch surface

$$
B=\mathbb{F}_{r} .
$$

The homology group of $\mathbb{F}_{r}$ has a basis of effective classes $\mathcal{S}$ and $\mathcal{E}$ with intersection numbers

$$
\mathcal{S}^{2}=-r, \quad \mathcal{S} \cdot \mathcal{E}=1, \quad \mathcal{E}^{2}=0
$$

The first and the second Chern classes of the tangent bundle of $\mathbb{F}_{r}$ are given by

$$
c_{1}\left(\mathbb{F}_{r}\right)=2 \mathcal{S}+(r+2) \mathcal{E}, \quad c_{2}\left(\mathbb{F}_{r}\right)=4 .
$$


Then, in general, $\mathcal{C}$ is given by (2.4) with

$$
\eta=a \mathcal{S}+b \mathcal{E},
$$

where $a$ and $b$ are integers. The class $\eta$ is effective if and only if

$$
a \geq 0, \quad b \geq 0 .
$$

The linear system $|\eta|$ is base point free if and only if [26]

$$
b \geq a r .
$$

Finally, $\eta-n c_{1}(B)$ is effective if and only if

$$
a \geq 2 n, \quad b \geq n(r+2) .
$$

Eqs. (2.11) and (2.12) guarantee that we can choose $\mathcal{C}$ to be an irreducible surface. In addition to the spectral cover, it is necessary to specify a line bundle, $\mathcal{N}$, over $\mathcal{C}$. In this paper, we will consider the case when, topologically, such a line bundle is a restriction of a global line bundle on $X$ (which we again denote by $\mathcal{N}$ ). For $S U(n)$ bundles, the first Chern class of $\mathcal{N}$ is given by $[8]$

$$
c_{1}(\mathcal{N})=n\left(\frac{1}{2}+\lambda\right) \sigma+\left(\frac{1}{2}-\lambda\right) \pi^{*} \eta+\left(\frac{1}{2}+n \lambda\right) \pi^{*} c_{1}(B) .
$$

Since $c_{1}(\mathcal{N})$ must be an integer class, it follows that either

$$
\lambda=m+\frac{1}{2} \quad \text { if } n \text { is odd }
$$

or

$$
\lambda=m, \quad \eta=c_{1}(B) \bmod 2 \text { if } n \text { is even, }
$$

where $m \in \mathbb{Z}$.

Given these ingredients, the $S U(n)$ vector bundle $V$ can be obtained by the Fourier-Mukai transform

$$
V=\pi_{1 *}\left(\pi_{2}^{*} \mathcal{N} \otimes \mathcal{P}\right) .
$$

Here $\pi_{1}$ and $\pi_{2}$ are the projections of the fiber product $X \times_{B} \mathcal{C}$ onto the two factors $X$ and $\mathcal{C}$ respectively. $\mathcal{P}$ is the Poincare line bundle. See 8 for details. The Chern classes of $V$ were computed in [8, 58, 59]. The results are

$$
\begin{aligned}
& c_{1}(V)=0, \\
& c_{2}(V)=\sigma \cdot \pi^{*} \eta-\frac{1}{24}\left(n^{3}-n\right) \pi^{*} c_{1}(B)+\frac{1}{2}\left(\lambda^{2}-\frac{1}{4}\right) n \pi^{*}\left(\eta \cdot\left(\eta-n c_{1}(B)\right)\right), \\
& c_{3}(V)=2 \lambda \sigma \cdot \pi^{*}\left(\eta \cdot\left(\eta-n c_{1}(B)\right)\right) .
\end{aligned}
$$


Finally, we note from the index theorem that

$$
N_{g e n}=\frac{c_{3}(V)}{2}
$$

gives the index of the Dirac operator in the fundamental representation of $S U(n)$ on a CalabiYau threefold and, therefore, the number of generations in the four-dimensional effective theory.

\subsection{Anomaly Cancellation and Five-Branes}

Any physically acceptable heterotic vacuum must be anomaly free. In the strongly coupled theory, this condition is given by [1]

$$
c_{2}\left(V_{1}\right)+c_{2}\left(V_{2}\right)+[W]=c_{2}(X)
$$

where $V_{1}$ and $V_{2}$ are the vector bundles on the observable and hidden orbifold fixed planes and $[W]$ is the class of the holomorphic curve in $X$ around which five-branes may be wrapped. In this paper, for simplicity, we will assume that the vector bundle in the hidden sector, $V_{2}$, is trivial. This simplifies eq. (2.21) to

$$
[W]=c_{2}(X)-c_{2}(V)
$$

where we denoted $V_{1}$ by $V$. This equation can be considered as a definition of the five-brane class $[W]$. Obviously, the five-brane class must, on physics grounds, be represented by an actual curve in $X$. Hence, $[W]$ must be an effective class in $H_{2}(X, \mathbb{Z})$. This condition puts a non-trivial restriction on the allowed vector bundle $V$. In the case of elliptically fibered Calabi-Yau threefolds, $[W]$ always decomposes as

$$
[W]=\sigma \cdot W_{B}+a_{f} F
$$

where $W_{B}=\pi^{*} w$ is the lift of a curve $w$ in the base, $a_{f}$ is an integer and $F$ is the class of the fiber. $[W]$ will be effective if and only if $w$ is effective and $a_{f}>0$.

\subsection{Examples of Models with Three Generations}

Let us give some examples of the above concepts. We will concentrate on GUT models having three generations of quarks and leptons. 
Example 1. Consider a vector bundle specified by $B=\mathbb{F}_{1}, G=S U(3)$ and the spectral cover

$$
\mathcal{C} \in\left|3 \sigma+\pi^{*}(6 \mathcal{S}+10 \mathcal{E})\right| .
$$

Clearly conditions (2.10)-(2.12) are satisfied. Let the parameter $\lambda$ of the line bundle $\mathcal{N}$ be

$$
\lambda=\frac{1}{2} .
$$

Using eqs. (2.2), (2.7), (2.8), (2.18) and (2.22), we find that the five-brane class is given by

$$
[W]=\sigma \cdot \pi^{*}(18 \mathcal{S}+26 \mathcal{E})+96 F .
$$

Since the curve $18 \mathcal{S}+26 \mathcal{E}$ is effective and 96 is non-negative, it follows that $[W]$ is effective. From (2.7), (2.19) we find

$$
N_{\text {gen }}=\frac{1}{2} c_{3}(V)=3 .
$$

Example 2. Consider a vector bundle specified by $B=\mathbb{F}_{1}, G=S U(3)$ and the spectral cover

$$
\mathcal{C} \in\left|3 \sigma+\pi^{*}(8 \mathcal{S}+9 \mathcal{E})\right| .
$$

Conditions (2.10)-(2.12) are satisfied. Choose the parameter $\lambda$ of the line bundle $\mathcal{N}$ be

$$
\lambda=\frac{3}{2} .
$$

From eqs. (2.2), (2.7), (2.8), (2.18) and (2.22) it follows that the five-brane class is given by

$$
[W]=\sigma \cdot \pi^{*}(16 \mathcal{S}+27 \mathcal{E})+90 F .
$$

Note that $[W]$ is effective. Using (2.7), (2.19) one finds

$$
N_{\text {gen }}=\frac{1}{2} c_{3}(V)=3 .
$$

We will refer to these examples later in the paper.

\section{Instanton Moduli}

\subsection{General Considerations}

The number of moduli of a stable, holomorphic $S U(n)$ vector bundle $V$ constructed using the Fourier-Mukai transform (2.16) is determined by the number of parameters specifying 
its spectral cover $\mathcal{C}$ and by the dimension of the space of holomorphic line bundles $\mathcal{N}$ on $\mathcal{C}$. In [27, it was shown that the number of parameters of the spectral cover $\mathcal{C}$ is given by the dimension of the linear system $|\mathcal{C}|$ in $X$. The linear system $|\mathcal{C}|$ is the projectivization of $H^{0}\left(X, \mathcal{O}_{X}(\mathcal{C})\right)$, the space of holomorphic sections of the line bundle $\mathcal{O}_{X}(\mathcal{C})$. That is

$$
|\mathcal{C}|=\mathbb{P} H^{0}\left(X, \mathcal{O}_{X}(\mathcal{C})\right)
$$

Therefore,

$$
\operatorname{dim}|\mathcal{C}|=h^{0}\left(X, \mathcal{O}_{X}(\mathcal{C})\right)-1
$$

This quantity counts the number of parameters specifying the spectral cover $\mathcal{C}$. The set of holomorphic line bundles $\mathcal{N}$ over $\mathcal{C}$, denoted by $\operatorname{Pic}(\mathcal{C})$, is, by definition, determined by the set of holomorphic transition functions on $\mathcal{C}$. A standard result (see, for example, 60] ) says that

$$
\operatorname{dim} \operatorname{Pic}(\mathcal{C})=h^{1}\left(\mathcal{C}, \mathcal{O}_{\mathcal{C}}\right)
$$

Putting this all together, we see that the number of moduli of a stable, holomorphic $S U(n)$ vector bundle $V$ is given by 27 ]

$$
n(V)=\left(h^{0}\left(X, \mathcal{O}_{X}(\mathcal{C})\right)-1\right)+h^{1}\left(\mathcal{C}, \mathcal{O}_{\mathcal{C}}\right)
$$

Since it will be important later in this paper, let us recall the argument for the identification (3.3). It follows from the exponential sequence on $\mathcal{C}$

$$
0 \rightarrow \mathbb{Z} \rightarrow \mathcal{O}_{\mathcal{C}} \rightarrow \mathcal{O}_{\mathcal{C}}^{*} \rightarrow 0
$$

where $\mathcal{O}_{\mathcal{C}}^{*}$ is the sheaf of holomorphic nowhere vanishing functions, that

$$
0 \rightarrow H^{1}(\mathcal{C}, \mathbb{Z}) \rightarrow H^{1}\left(\mathcal{C}, \mathcal{O}_{\mathcal{C}}\right) \rightarrow \operatorname{Pic}^{0}(\mathcal{C}) \rightarrow 0
$$

Here $P i c^{0}(\mathcal{C})$ describes the space of all degree zero line bundles on $\mathcal{C}$. Since $H^{1}(\mathcal{C}, \mathbb{Z})$ is a discrete lattice we find $\operatorname{dim} \operatorname{Pic}^{0}(\mathcal{C})=h^{1}\left(\mathcal{C}, \mathcal{O}_{\mathcal{C}}\right)$. In addition, since $\operatorname{dim} \operatorname{Pic}^{0}(\mathcal{C})=\operatorname{dim} \operatorname{Pic}(\mathcal{C})$, identification (3.3) follows. On a Calabi-Yau threefold $X$, we can describe the vector space $H^{1}\left(\mathcal{C}, \mathcal{O}_{\mathcal{C}}\right)$ rather explicitly. Considering the exact sequence

$$
0 \rightarrow \mathcal{O}_{X}(-\mathcal{C}) \rightarrow \mathcal{O}_{X} \rightarrow \mathcal{O}_{\mathcal{C}} \rightarrow 0
$$

and the corresponding cohomology sequence

$$
\cdots \rightarrow H^{1}\left(X, \mathcal{O}_{X}\right) \rightarrow H^{1}\left(\mathcal{C}, \mathcal{O}_{\mathcal{C}}\right) \rightarrow H^{2}\left(X, \mathcal{O}_{X}(-\mathcal{C})\right) \rightarrow H^{2}\left(X, \mathcal{O}_{X}\right) \rightarrow \ldots
$$


we obtain

$$
H^{1}\left(\mathcal{C}, \mathcal{O}_{\mathcal{C}}\right)=H^{2}\left(X, \mathcal{O}_{X}(-\mathcal{C})\right)
$$

Here we have used

$$
H^{i}\left(X, \mathcal{O}_{X}\right)=H_{\bar{\partial}}^{0, i}(X)
$$

together with the fact that on a Calabi-Yau manifold $X$

$$
h^{0,1}=h^{0,2}=0
$$

Furthermore, by Serre duality,

$$
H^{2}\left(X, \mathcal{O}_{X}(-\mathcal{C})\right)=H^{1}\left(X, \mathcal{O}_{X}(\mathcal{C})\right)^{*}
$$

Hence, we find

$$
H^{1}\left(\mathcal{C}, \mathcal{O}_{\mathcal{C}}\right)=H^{1}\left(X, \mathcal{O}_{X}(\mathcal{C})\right)^{*}
$$

and, in particular, that

$$
h^{1}\left(\mathcal{C}, \mathcal{O}_{\mathcal{C}}\right)=h^{1}\left(X, \mathcal{O}_{X}(\mathcal{C})\right)
$$

Therefore, eq. (3.4) can be rewritten as

$$
n(V)=\left(h^{0}\left(X, \mathcal{O}_{X}(\mathcal{C})\right)-1\right)+h^{1}\left(X, \mathcal{O}_{X}(\mathcal{C})\right)
$$

This expression suggests that in order to calculate the number of moduli, one has to learn how to calculate the dimension of the sheaf cohomology groups $h^{0}\left(X, \mathcal{O}_{X}(\mathcal{C})\right)$ and $h^{1}\left(X, \mathcal{O}_{X}(\mathcal{C})\right)$. In this paper, for specificity, we will concentrate on Calabi-Yau threefolds elliptically fibered over Hirzebruch surfaces $\mathbb{F}_{r}$ as we did in [27]. We will also choose the index $r$ to be $0,1,2$. The Calabi-Yau threefolds fibered over $\mathbb{F}_{r}$ for $r>3$ are singular and require a different approach. In [27, the problem of calculating the dimension of the above sheaf cohomologies was simplified by imposing a special constraint on the spectral cover, called positivity. We will review this in the next subsection. However, as we will see, most interesting GUT models with three generations involve vector bundles whose spectral covers are not positive. This is the case, for instance, in both examples in Section 2. In this paper, we will improve the results of [27] by giving expressions for the number of vector bundle moduli in vacua where the spectral cover is not positive. 


\subsection{Review of Bundles with Positive Spectral Cover}

The simplifying condition on the spectral cover imposed in [27] was positivity. By definition, $\mathcal{C}$ is positive if the first Chern class of the associated line bundle $\mathcal{O}_{X}(\mathcal{C})$ can be represented by a positive definite two-form. Equivalently, $\mathcal{C}$ is positive if

$$
\mathcal{C} \cdot z>0
$$

for every irreducible, effective curve $z$ in $X$. In [27, it was shown that, for Calabi-Yau threefolds elliptically fibered over Hirzebruch surfaces, the positivity condition imposes the following restrictions on the coefficients $a$ and $b$ in (2.4):

$$
\begin{aligned}
& a>2 n, \\
& b>a r+n(2-r) .
\end{aligned}
$$

Subject to this condition, the calculation of $n(V)$ is greatly simplified. It was shown in [27] that, in this case,

$$
h^{i}\left(X, \mathcal{O}_{X}(\mathcal{C})\right)=0, \quad i>0
$$

As a consequence, $h^{0}\left(X, \mathcal{O}_{X}(\mathcal{C})\right)$ is equal to the Euler characteristic $\chi_{E}\left(X, \mathcal{O}_{X}(\mathcal{C})\right)$ which can be computed using the index theorem

$$
\chi_{E}\left(X, \mathcal{O}_{X}(\mathcal{C})\right)=\int_{X} \operatorname{ch}\left(\mathcal{O}_{X}(\mathcal{C})\right) \wedge T d(X)
$$

The direct evaluation of the right hand side of (3.19) gives (see [27] for details)

$$
\chi_{E}\left(X, \mathcal{O}_{X}(\mathcal{C})\right)=\frac{n}{3}\left(4 n^{2}-1\right)+n a b-\left(n^{2}-2\right)(a+b)+a r\left(\frac{n^{2}}{2}-1\right)-\frac{n}{2} r a^{2}
$$

and, therefore,

$$
n(V)=\frac{n}{3}\left(4 n^{2}-1\right)+n a b-\left(n^{2}-2\right)(a+b)+\operatorname{ar}\left(\frac{n^{2}}{2}-1\right)-\frac{n}{2} r a^{2}-1 .
$$

The expression (3.20) for $\chi_{E}\left(X, \mathcal{O}_{X}(\mathcal{C})\right)$ is valid for all choices of $a$ and $b$, whereas the expression for $n(V)$ (3.21) is valid only if inequalities (3.17) are satisfied. However, it is straightforward to check that in the three families examples presented in Section 2, the integers $a$ and $b$ do not satisfy eq. (3.17). Thus, unfortunately, many physically interesting vacua do not arise from bundles with positive spectral cover. 


\subsection{Bundles with Arbitrary Irreducible Spectral Cover}

In this subsection, the positivity condition will not be imposed. Therefore, we have to find an alternative procedure for calculating $h^{0}\left(X, \mathcal{O}_{X}(\mathcal{C})\right)$ and $h^{1}\left(X, \mathcal{O}_{X}(\mathcal{C})\right)$. For specificity, we consider Calabi-Yau threefolds elliptically fibered over $\mathbb{F}_{r}$. Let us recall the structure of $\mathbb{F}_{r}$. The Hirzebruch surface $\mathbb{F}_{r}$ is a $\mathbb{P}^{1}$ bundle over $\mathbb{P}^{1}, \mathbb{F}_{0}$ being just the trivial product $\mathbb{P}^{1} \times \mathbb{P}^{1}$. The base of this bundle is the curve $\mathcal{S} \simeq \mathbb{P}^{1}$ and the fiber is the curve $\mathcal{E} \simeq \mathbb{P}^{1}$. Their intersection numbers are given in eq. (2.7). Any spectral cover will be of the form

$$
\mathcal{C} \in\left|n \sigma+\pi^{*} \eta\right|
$$

where

$$
\eta=a \mathcal{S}+b \mathcal{E}
$$

and the coefficients $a$ and $b$ satisfy eqs. (2.10)-(2.12). Recall that $n$ is the rank of the vector bundle. Let us denote the projection of $\mathbb{F}_{r}$ onto the base $\mathcal{S}$ by $\rho$

$$
\rho: B=\mathbb{F}_{r} \rightarrow \mathcal{S}
$$

The natural idea will be to relate $h^{0}\left(X, \mathcal{O}_{X}(\mathcal{C})\right)$ and $h^{1}\left(X, \mathcal{O}_{X}(\mathcal{C})\right)$ to cohomology groups on $\mathcal{S} \simeq \mathbb{P}^{1}$ and then use the standard expressions

$$
h^{0}\left(\mathbb{P}^{1}, \mathcal{O}_{\mathbb{P}^{1}}(m)\right)=m+1, \quad m \geq 0
$$

and

$$
h^{0}\left(\mathbb{P}^{1}, \mathcal{O}_{\mathbb{P}^{1}}(m)\right)=0, \quad m \leq-1
$$

as well as

$$
h^{1}\left(\mathbb{P}^{1}, \mathcal{O}_{\mathbb{P}^{1}}(m)\right)=0, \quad m \geq 0
$$

and

$$
h^{1}\left(\mathbb{P}^{1}, \mathcal{O}_{\mathbb{P}^{1}}(m)\right)=-m-1, \quad m \leq-1 .
$$

By definition

$$
H^{0}\left(X, \mathcal{O}_{X}(\mathcal{C})\right)=H^{0}\left(B, \pi_{*} \mathcal{O}_{X}(\mathcal{C})\right)
$$

Using a Leray spectral sequence, we find

$$
H^{1}\left(X, \mathcal{O}_{X}(\mathcal{C})\right)=H^{1}\left(B, \pi_{*} \mathcal{O}_{X}(\mathcal{C})\right)
$$

To justify (3.30), we have to show that

$$
H^{0}\left(B, R^{1} \pi_{*} \mathcal{O}_{X}(\mathcal{C})\right)=0
$$


This can be seen from the following simple calculation. By definition, for every point $p$ in the base $B=\mathbb{F}_{r}$ we have

$$
\left.R^{1} \pi_{*} \mathcal{O}_{X}(\mathcal{C})\right|_{p \in B}=H^{1}\left(F_{p},\left.\mathcal{O}_{X}(\mathcal{C})\right|_{F_{p}}\right)
$$

where $F_{p}$ is the elliptic fiber over $p$. For a spectral cover of the form (3.22), (3.23) we have

$$
\mathcal{O}_{F_{p}}\left(\left.\mathcal{C}\right|_{F_{p}}\right)=\mathcal{O}_{F_{p}}\left(\left(n \sigma+\pi^{*}(a \mathcal{S}+b \mathcal{E})\right) \cdot F\right)=\mathcal{O}_{F_{p}}(n \sigma(p)) .
$$

Here, we have used the intersection properties

$$
\sigma \cdot F=\sigma(p), \quad \pi^{*} \mathcal{S} \cdot F=\pi^{*} \mathcal{E} \cdot F=0
$$

On any irreducible curve, $H^{1}$ with coefficients in any line bundle of positive degree vanishes

$$
H^{1}\left(F_{p}, \mathcal{O}_{F_{p}}(n \sigma(p))\right)=0
$$

We will assume in this paper that all fibers of $\pi$ are irreducible. Then, from eq. (3.32) we conclude that the sheaf $R^{1} \pi_{*} \mathcal{O}_{X}(\mathcal{C})$ is the zero sheaf,

$$
R^{1} \pi_{*} \mathcal{O}_{X}(\mathcal{C})=0
$$

since it vanishes at every point $p \in B$. This proves eq. (3.31) and, hence, eq. (3.30).

To proceed, we need to calculate $\pi_{*} \mathcal{O}_{X}(\mathcal{C})$. Since

$$
\pi_{*} \mathcal{O}_{X}(\mathcal{C})=\pi_{*} \mathcal{O}_{X}\left(n \sigma+\pi^{*} \eta\right)=\pi_{*} \mathcal{O}_{X}(n \sigma) \otimes \mathcal{O}_{B}(\eta)
$$

it suffices to calculate $\pi_{*} \mathcal{O}_{X}(n \sigma)$. This can be done by induction. Take $n=1$ and consider the following sequence

$$
\left.0 \rightarrow \mathcal{O}_{X} \rightarrow \mathcal{O}_{X}(\sigma) \rightarrow \mathcal{O}_{X}(\sigma)\right|_{\sigma} \rightarrow 0
$$

Inclusion $\mathcal{O}_{X} \hookrightarrow \mathcal{O}_{X}(\sigma)$ induces the sheaf map

$$
i: \pi_{*} \mathcal{O}_{X} \rightarrow \pi_{*} \mathcal{O}_{X}(\sigma)
$$

Restricted to any point $p \in B$, this map becomes

$$
i_{p}: H^{0}\left(F_{p}, \mathcal{O}_{F_{p}}\right) \rightarrow H^{0}\left(F_{p},\left.\mathcal{O}_{F_{p}}(\sigma)\right|_{F_{p}}\right)
$$

where we have used the fact that $\pi_{*} \mathcal{O}_{X}=\mathcal{O}_{B}$. Since $\sigma \cdot F=1$, the degree of $\left.\mathcal{O}_{F_{p}}(\sigma)\right|_{F_{p}}$ is unity and, hence, it has a unique holomorphic section. Then the map $i_{p}$ is an isomorphism. Since this is true at any point $p \in B$, it follows that

$$
\pi_{*} \mathcal{O}_{X}(\sigma)=\pi_{*} \mathcal{O}_{X}=\mathcal{O}_{B}
$$


Now take $n=2$ and consider the sequence

$$
\left.0 \rightarrow \mathcal{O}_{X}(\sigma) \rightarrow \mathcal{O}_{X}(2 \sigma) \rightarrow \mathcal{O}_{X}(2 \sigma)\right|_{\sigma} \rightarrow 0
$$

Using (3.41), the relation

$$
\sigma \cdot \sigma=-\pi^{*} c_{1}(B) \cdot \sigma
$$

and the fact that

$$
R^{1} \pi_{*} \mathcal{O}_{X}(\sigma)=0
$$

the direct image of the sequence (3.42) can be expressed as

$$
0 \rightarrow \mathcal{O}_{B} \rightarrow \pi_{*} \mathcal{O}_{X}(2 \sigma) \rightarrow \mathcal{O}_{B}\left(-2 c_{1}(B)\right) \rightarrow 0
$$

Relation (3.43) was proven in [8, whereas (3.44) follows as a special case of eq. (3.31) with $a=b=0$. From eq. (3.45), we would like to conclude that

$$
\pi_{*} \mathcal{O}_{X}(2 \sigma)=\mathcal{O}_{B} \oplus \mathcal{O}_{B}\left(-2 c_{1}(B)\right)
$$

This is indeed the case provided the extension group

$$
\operatorname{Ext}_{B}^{1}\left(\mathcal{O}_{B}\left(-2 c_{1}(B)\right), \mathcal{O}_{B}\right) \simeq H^{1}\left(B, \mathcal{O}_{B}\left(2 c_{1}(B)\right)\right)=0
$$

We will prove this below for the relevant case of $B=\mathbb{F}_{r}, r=0,1,2$. Continuing in this way, we obtain

$$
\pi_{*} \mathcal{O}_{X}(n \sigma)=\mathcal{O}_{B} \oplus \bigoplus_{i=2}^{n} \mathcal{O}_{B}\left(-i c_{1}(B)\right) .
$$

It follows from this and (3.37) that

$$
\pi_{*} \mathcal{O}_{X}(\mathcal{C})=\mathcal{O}_{B}(\eta) \oplus \bigoplus_{i=2}^{n} \mathcal{O}_{B}\left(\eta-i c_{1}(B)\right)
$$

In the previous section, we stated that the stability of the bundle demands that $\eta-n c_{1}(B)$ be effective. It follows that every line bundle on the right hand side of eq. (3.49) must be of the form

$$
\mathcal{O}_{B}(c \mathcal{S}+d \mathcal{E}), \quad c \geq 0, \quad d \geq 0
$$

We now use the second projection $\rho$ given by eq. (3.24) to relate data on $B=\mathbb{F}_{r}$ to data on $\mathcal{S} \simeq \mathbb{P}_{1}$. Note that by definition it follows that

$$
H^{0}\left(B, \mathcal{O}_{B}(c \mathcal{S}+d \mathcal{E})\right)=H^{0}\left(\mathcal{S}, \rho_{*} \mathcal{O}_{B}(c \mathcal{S}+d \mathcal{E})\right)
$$


and from a Leray spectral sequence argument, that

$$
H^{1}\left(B, \mathcal{O}_{B}(c \mathcal{S}+d \mathcal{E})\right)=H^{1}\left(\mathcal{S}, \rho_{*} \mathcal{O}_{B}(c \mathcal{S}+d \mathcal{E})\right) .
$$

In eq. (3.52), we have used the fact that

$$
R^{1} \rho_{*} \mathcal{O}_{B}(c \mathcal{S}+d \mathcal{E})=0, \quad c, d \geq 0 .
$$

This can be proven is a similar way to eq. (3.36). Combining these results with (3.29), (3.30), (3.49) and (3.50), we have

$$
H^{0}\left(X, \mathcal{O}_{X}(\mathcal{C})\right)=H^{0}\left(\mathcal{S}, \rho_{*} \pi_{*} \mathcal{O}_{X}(\mathcal{C})\right)
$$

and

$$
H^{1}\left(X, \mathcal{O}_{X}(\mathcal{C})\right)=H^{1}\left(\mathcal{S}, \rho_{*} \pi_{*} \mathcal{O}_{X}(\mathcal{C})\right) .
$$

Therefore, by computing $\rho_{*} \mathcal{O}_{B}(c \mathcal{S}+d \mathcal{E})$ for $c, d \geq 0$, one can relate the cohomology groups $H^{0}\left(X, \mathcal{O}_{X}(\mathcal{C})\right)$ and $H^{1}\left(X, \mathcal{O}_{X}(\mathcal{C})\right)$ to cohomology groups on the curve $\mathcal{S}$ by means of eqs. (3.54) and (3.55). We begin by noting that

$$
\rho_{*} \mathcal{O}_{B}(c \mathcal{S}+d \mathcal{E})=\rho_{*} \mathcal{O}_{B}(c \mathcal{S}) \otimes \mathcal{O}_{\mathcal{S}}(d) .
$$

Hence, it suffices to compute $\rho_{*} \mathcal{O}_{B}(c \mathcal{S})$ for $c \geq 0$. This can be accomplished by an induction procedure similar to that used for $\pi_{*} \mathcal{O}_{X}(n \sigma)$. Obviously, for $c=0$

$$
\rho_{*} \mathcal{O}_{B}=\mathcal{O}_{\mathcal{S}}
$$

Take $c=1$ and consider the short exact sequence

$$
\left.0 \rightarrow \mathcal{O}_{B} \rightarrow \mathcal{O}_{B}(\mathcal{S}) \rightarrow \mathcal{O}_{B}(\mathcal{S})\right|_{\mathcal{S}} \rightarrow 0 .
$$

Taking its direct image, we get

$$
0 \rightarrow \mathcal{O}_{\mathcal{S}} \rightarrow \rho_{*} \mathcal{O}_{B}(\mathcal{S}) \rightarrow \mathcal{O}_{\mathcal{S}}(-r) \rightarrow 0,
$$

where $r$ is the subscript of $\mathbb{F}_{r}$ and we have used eqs. (2.7) and (3.53). One can easily show that

$$
\operatorname{Ext}_{\mathbb{P}^{1}}^{1}\left(\mathcal{O}_{\mathcal{S}}(-r), \mathcal{O}_{\mathcal{S}}\right)=H^{1}\left(\mathbb{P}^{1}, \mathcal{O}_{\mathcal{S}}(r)\right)=0
$$

for positive $r$ Hence, we find

$$
\rho_{*} \mathcal{O}_{B}(\mathcal{S})=\mathcal{O}_{\mathcal{S}} \oplus \mathcal{O}_{\mathcal{S}}(-r) .
$$


Now take $c=2$ and consider the sequence

$$
\left.0 \rightarrow \mathcal{O}_{B}(\mathcal{S}) \rightarrow \mathcal{O}_{B}(2 \mathcal{S}) \rightarrow \mathcal{O}_{B}(2 \mathcal{S})\right|_{\mathcal{S}} \rightarrow 0
$$

Taking the direct image, and showing that the corresponding extension space vanishes, we get

$$
0 \rightarrow \mathcal{O}_{\mathcal{S}} \oplus \mathcal{O}_{\mathcal{S}}(-r) \rightarrow \rho_{*} \mathcal{O}_{B}(2 \mathcal{S}) \rightarrow \mathcal{O}_{\mathcal{S}}(-2 r) \rightarrow 0
$$

where eqs. (3.53) and (3.61) have been used. Then

$$
\rho_{*} \mathcal{O}_{B}(2 \mathcal{S})=\mathcal{O}_{\mathcal{S}} \oplus \mathcal{O}_{\mathcal{S}}(-r) \oplus \mathcal{O}_{\mathcal{S}}(-2 r)
$$

Continuing in this way, we obtain

$$
\rho_{*} \mathcal{O}_{B}(c \mathcal{S})=\mathcal{O}_{\mathcal{S}} \oplus \bigoplus_{j=1}^{c} \mathcal{O}_{\mathcal{S}}(-j r)
$$

It follows from this and (3.56) that

$$
\rho_{*} \mathcal{O}_{B}(c \mathcal{S}+d \mathcal{E})=\mathcal{O}_{\mathcal{S}}(d) \oplus \bigoplus_{j=1}^{c} \mathcal{O}_{\mathcal{S}}(d-j r)
$$

Eqs. (3.49), (3.54), (3.55) and (3.66) provide a complete relation between data on $X$ and data on $\mathbb{P}^{1}$. We will use them to calculate $h^{0}\left(X, \mathcal{O}_{X}(\mathcal{C})\right)$ and $h^{1}\left(X, \mathcal{O}_{X}(\mathcal{C})\right)$.

First, however, we will prove (3.47). From (2.8),

$$
H^{1}\left(B, \mathcal{O}_{B}\left(2 c_{1}(B)\right)\right)=H^{1}\left(B, \mathcal{O}_{B}(4 \mathcal{S}+2(r+2) \mathcal{E})\right)
$$

Using eqs. (3.52) and (3.66), it follows that

$$
\begin{aligned}
& H^{1}\left(B, \mathcal{O}_{B}(4 \mathcal{S}+2(r+2) \mathcal{E})\right)=H^{1}\left(\mathcal{S}, \rho_{*} \mathcal{O}_{B}(4 \mathcal{S}+2(r+2) \mathcal{E})\right)= \\
& H^{1}\left(\mathcal{S}, \mathcal{O}(2(r+2)) \oplus \bigoplus_{j=1}^{4} \mathcal{O}_{\mathcal{S}}(2(r+2)-j r)\right)
\end{aligned}
$$

Note that in the last expression each line bundle on $\mathcal{S}$ has non-negative degree for $r=0,1,2$. Therefore, by eqs. (3.28) and (3.27), each has vanishing $H^{1}$. This proves (3.47).

We now have all ingredients necessary to compute the number of moduli for a vector bundle specified by a spectral cover

$$
\mathcal{C} \in\left|n \sigma+\pi^{*}(a \mathcal{S}+b \mathcal{E})\right|
$$


Recall, that the conditions on the coefficients $a$ and $b$ are

$$
a, b \geq 0, \quad b \geq a r, \quad a \geq 2 n, \quad b \geq n(r+2) .
$$

We need to compute the form of the line bundles that arise in $\rho_{*} \pi_{*} \mathcal{O}_{X}(\mathcal{C})$. From eqs. (3.49), (2.8) and (3.69) we have

$$
\pi_{*} \mathcal{O}_{X}(\mathcal{C})=\mathcal{O}_{B}(a \mathcal{S}+b \mathcal{E}) \oplus \bigoplus_{i=2}^{n} \mathcal{O}_{B}((a-2 i) \mathcal{S}+(b-(r+2) i) \mathcal{E})
$$

Now consider the $\rho$-direct image of all the line bundles on the right hand side of (3.71). Using (3.66), we see that

$$
\rho_{*} \mathcal{O}_{B}(a \mathcal{S}+b \mathcal{E})=\mathcal{O}_{\mathcal{S}}(b) \oplus \bigoplus_{j=1}^{a} \mathcal{O}_{\mathcal{S}}(b-j r)
$$

Note from (3.70) that all degrees on the right hand side of (3.72) are non-negative. Similarly, (3.66) implies

$$
\rho_{*} \mathcal{O}_{B}\left((a-2 i) \mathcal{S}+(b-(r+2) i \mathcal{E})=\mathcal{O}_{\mathcal{S}}(b-(r+2) i) \oplus \bigoplus_{j=1}^{a-2 i} \mathcal{O}_{\mathcal{S}}(b-(r+2) i-j r)\right.
$$

It is easy to see that if $r=0,2$, all degrees on the right hand side of eq. (3.73) are nonnegative as a consequence of eq. (3.70). Indeed, if $r=0$, all degrees in eq. (3.73) are $b-2 i$. Since $i$ runs from 2 to $n$, the minimal possible degree is $b-2 n$. But from eq. (3.70) we see that $b$ is always greater or equal $2 n$. Similarly, when $r=2$, the minimal possible degree in (3.73) is when $j$ is maximal, that is, $b-4 i-2(a-2 i)=b-2 a$. But from eq. (3.70) we see that $b-2 a$ is always non-negative for $r=2$. On the contrary, for $r=1, \rho_{*} \pi_{*} \mathcal{O}_{X}(\mathcal{C})$ contains line bundles of both positive and negative degree. We find it convenient, therefore, to separate the cases $r=0,2$ and $r=1$. In the first case, the calculation of $h^{0}\left(X, \mathcal{O}_{X}(\mathcal{C})\right)$ and $h^{1}\left(X, \mathcal{O}_{X}(\mathcal{C})\right)$ will be relatively easy, whereas in the latter case it will require greater care.

$r=0,2$ case:

Let us begin with the case $r=0,2$. We have just concluded that, in this case, $\rho_{*} \pi_{*} \mathcal{O}_{X}(\mathcal{C})$ contains only line bundles on $\mathcal{S}$ of non-negative degree. Then from eqs. (3.28) and (3.27) it follows that

$$
h^{1}\left(X, \mathcal{O}_{X}(\mathcal{C})\right)=0
$$


That is, as in the case of bundles with positive spectral cover, the line bundles $\mathcal{N}$ do not have any continuous parameters. Now calculate $h^{0}\left(X, \mathcal{O}_{X}(\mathcal{C})\right.$ ). Using (2.8), (3.29), and (3.49), we have

$$
h^{0}\left(X, \mathcal{O}_{X}(\mathcal{C})\right)=h^{0}\left(B, \mathcal{O}_{B}(a \mathcal{S}+b \mathcal{E})\right)+\sum_{i=2}^{n} h^{0}\left(B, \mathcal{O}_{B}((a-2 i) \mathcal{S}+(b-(r+2) i) \mathcal{E})\right)
$$

Consider the first term. By eq. (3.72)

$$
h^{0}\left(B, \mathcal{O}_{B}(a \mathcal{S}+b \mathcal{E})\right)=h^{0}\left(\mathcal{S}, \mathcal{O}_{\mathcal{S}}(b)\right)+\sum_{j=1}^{a} h^{0}\left(\mathcal{S}, \mathcal{O}_{\mathcal{S}}(b-r j)\right)
$$

Since all degree in eq. (3.76) are positive, it follows from from (3.25) that

$$
h^{0}\left(B, \mathcal{O}_{B}(a \mathcal{S}+b \mathcal{E})\right)=(b+1)+\sum_{j=1}^{a}(b-r j+1)=(a+1)\left(b+1-\frac{r a}{2}\right) .
$$

Similarly,

$$
\begin{aligned}
& \sum_{i=2}^{n} h^{0}\left(B, \mathcal{O}_{B}((a-2 i) \mathcal{S}+(b-(r+2) i) \mathcal{E})\right)=\sum_{i=2}^{n} h^{0}\left(\mathcal{S}, \mathcal{O}_{\mathcal{S}}(b-(r+2) i)\right)+ \\
& \sum_{i=2}^{n} h^{0}\left(\mathcal{S}, \bigoplus_{j=1}^{a-2 i} \mathcal{O}_{\mathcal{S}}(b-(r+2) i-j r)\right) .
\end{aligned}
$$

Since all degrees here are non-negative, we find using eq. (3.25) that

$$
\begin{aligned}
& \sum_{i=2}^{n} h^{0}\left(B, \mathcal{O}_{B}((a-2 i) \mathcal{S}+(b-(r+2) i) \mathcal{E})\right)= \\
& \sum_{i=2}^{n}(b-(r+2) i+1)+\sum_{i=2}^{n} \sum_{j=1}^{a-2 i}(b-(r+2) i-j r+1)= \\
& \frac{1}{2}(n-1)(n r-2 n-2 r+2 b-2)+ \\
& \frac{1}{6}(n-1)\left(12+6 a+12 b-6 a b-14 n+6 a n+6 b n-8 n^{2}-6 r-3 a r+\right. \\
& \left.3 a^{2} r-3 n r-3 a n r\right) .
\end{aligned}
$$

Adding eqs. (3.77) and (3.79), we get

$$
h^{0}\left(X, \mathcal{O}_{X}(\mathcal{C})\right)=\frac{n}{3}\left(4 n^{2}-1\right)+n a b-\left(n^{2}-2\right)(a+b)+\operatorname{ar}\left(\frac{n^{2}}{2}-1\right)-\frac{n}{2} r a^{2} .
$$

Combining (3.15), (3.74) and (3.80), the number of moduli is given by

$$
n(V)=\frac{n}{3}\left(4 n^{2}-1\right)+n a b-\left(n^{2}-2\right)(a+b)+\operatorname{ar}\left(\frac{n^{2}}{2}-1\right)-\frac{n}{2} r a^{2}-1 .
$$


This is the final expression for the number of instanton moduli for $r=0,2$. Surprisingly, it coincides with eq. (3.21) obtained for bundles with positive spectral cover. In addition, eq. (3.80) coincides with eq. (3.20) for the Euler characteristic. That is

$$
h^{0}\left(X, \mathcal{O}_{X}(\mathcal{C})\right)=\chi_{E}\left(X, \mathcal{O}_{X}(\mathcal{C})\right)
$$

It follows that not only does $h^{1}\left(X, \mathcal{O}_{X}(\mathcal{C})\right)$ vanish, as we have shown, but

$$
h^{2}\left(X, \mathcal{O}_{X}(\mathcal{C})\right)=h^{3}\left(X, \mathcal{O}_{X}(\mathcal{C})\right)=0
$$

must be true as well. As a consistency check of our results, we will prove in Appendix A, using independent methods, that this is indeed the case.

$r=1$ case:

Let us now consider the more complicated case when $r=1$. In this case, $h^{1}\left(X, \mathcal{O}_{X}(\mathcal{C})\right)$ will generically be non-zero and line bundles on $\mathcal{C}$ will contribute instanton moduli. To begin with, we consider eq. (3.73). This will contain only line bundles of non-negative degree if (see eq. (3.27))

$$
b-3 i-j \geq-1
$$

for all allowed values of $i$ and $j$. Inequality (3.84) is satisfied if and only if

$$
b \geq a+n-1
$$

i) $b \geq a+n-1$ :

Assume that $a, b$ satisfy (3.85). Then it follows from (3.27) that $h^{1}\left(X, \mathcal{O}_{X}(\mathcal{C})\right)$ will not contribute to $n(V)$. In this case, the entire contribution to $n(V)$ comes from $h^{0}\left(X, \mathcal{O}_{X}(\mathcal{C})\right)$ as before. A calculation identical to that done in the case $r=0,2$ again leads eq. (3.81). We conclude that if

$$
r=1, \quad b \geq a+n-1,
$$

the number of moduli is given by

$$
n(V)=\frac{n}{3}\left(4 n^{2}-1\right)+n a b-\left(n^{2}-2\right)(a+b)+\operatorname{ar}\left(\frac{n^{2}}{2}-1\right)-\frac{n}{2} r a^{2}-1 .
$$

As before, $h^{0}\left(X, \mathcal{O}_{X}(\mathcal{C})\right)$ coincides with $\chi_{E}$. 
ii) $b<a+n-1$ :

Now assume that

$$
b<a+n-1 .
$$

Let us first calculate $h^{1}\left(X, \mathcal{O}_{X}(\mathcal{C})\right)$. From eq. (3.75),$\pi_{*} \mathcal{O}_{X}(\mathcal{C})$ contains two contributions

$$
\mathcal{O}_{B}(a \mathcal{S}+b \mathcal{E})
$$

and

$$
\left.\bigoplus_{i=2}^{n} \mathcal{O}_{B}((a-2 i) \mathcal{S}+(b-(r+2) i) \mathcal{E})\right)
$$

Using (3.72), the direct image with respect to $\rho$ of (3.89) is

$$
\rho_{*} \mathcal{O}_{B}(a \mathcal{S}+b \mathcal{E})=\mathcal{O}_{\mathcal{S}}(b) \oplus \bigoplus_{j=1}^{a} \mathcal{O}_{\mathcal{S}}(b-j)
$$

which does not contain line bundles of degree less than -1 . Hence, its contribution to $h^{1}\left(X, \mathcal{O}_{X}(\mathcal{C})\right)$ vanishes. Now consider the $\rho$-direct image of line bundles in (3.90). From eq. (3.73) we have

$$
\begin{aligned}
& \rho_{*} \mathcal{O}_{B}\left((a-2 i) \mathcal{S}+(b-3 i \mathcal{E})=\mathcal{O}_{\mathcal{S}}(b-3 i) \oplus \bigoplus_{j=1}^{a-2 i} \mathcal{O}_{\mathcal{S}}(b-3 i-j r)=\right. \\
& \mathcal{O}_{\mathcal{S}}(b-3 i) \oplus \mathcal{O}_{\mathcal{S}}(b-3 i-1) \oplus \cdots \oplus \mathcal{O}_{\mathcal{S}}(b-a-1)
\end{aligned}
$$

In order for $h^{1}\left(\mathcal{S}, \rho_{*} \mathcal{O}_{B}((a-2 i) \mathcal{S}+(b-3 i \mathcal{E}))\right)$ to be non-zero, at least one degree in eq. (3.92) must be less than -1 . For $i \leq b-a+1$, all degrees in eq. (3.92) are greater than -1 and

$$
h^{1}\left(\mathcal{S}, \rho_{*} \mathcal{O}_{B}((a-2 i) \mathcal{S}+(b-3 i \mathcal{E}))\right)=0 .
$$

For $i=b-a+2$, we have

$$
\rho_{*} \mathcal{O}_{B}((a-2 i) \mathcal{S}+(b-3 i \mathcal{E}))=\mathcal{O}_{\mathcal{S}}(-2) \oplus \mathcal{O}_{\mathcal{S}}(-1) \oplus \mathcal{O}_{\mathcal{S}} \oplus \ldots
$$

where the ellipsis denote the line bundles of positive degree. Therefore, by eqs. (3.28) and (3.27) we get

$$
h^{1}\left(\mathcal{S}, \rho_{*} \mathcal{O}_{B}((a-2 i) \mathcal{S}+(b-3 i \mathcal{E}))\right)=1 .
$$

More generally, for $b-a+1<i \leq n$ we have

$$
\rho_{*} \mathcal{O}_{B}((a-2 i) \mathcal{S}+(b-3 i \mathcal{E}))=\mathcal{O}_{\mathcal{S}}(b-a-i) \oplus \mathcal{O}_{\mathcal{S}}(b-a+1-i) \oplus \mathcal{O}_{\mathcal{S}}(-1) \oplus \mathcal{O}_{\mathcal{S}} \oplus \ldots
$$


where the ellipsis denote line bundles of positive degree. From eqs. (3.96), (3.28) and (3.27) we find

$$
h^{1}\left(\mathcal{S}, \rho_{*} \mathcal{O}_{B}((a-2 i) \mathcal{S}+(b-3 i \mathcal{E}))\right)=\sum_{j=2}^{i-b+a}(j-1)=\frac{(i-b+a)(i-b+a-1)}{2} .
$$

To find $h^{1}\left(X, \mathcal{O}_{X}(\mathcal{C})\right)$, we have to sum over $i$ from $b-a+2$ to $n$. The result is

$$
\begin{aligned}
& h^{1}\left(X, \mathcal{O}_{X}(\mathcal{C})\right)=\sum_{i=b-a+2}^{n} \frac{(i-b+a)(i-b+a-1)}{2}= \\
& \frac{1}{6}(n-b+a)(n-b+a-1)(n-b+a+1) .
\end{aligned}
$$

This concludes our calculation of $h^{1}\left(X, \mathcal{O}_{X}(\mathcal{C})\right)$. Similarly, we can compute $h^{0}\left(X, \mathcal{O}_{X}(\mathcal{C})\right)$. It follows from eqs. (3.25) and (3.26) that in the $\rho$-direct image of eqs. (3.89) and (3.90) only line bundles of non-negative degree will contribute. First,

$$
h^{0}\left(B, \mathcal{O}_{B}(a \mathcal{S}+b \mathcal{E})\right)=(a+1)\left(b+1-\frac{r a}{2}\right)
$$

as was computed previously in (3.77). Furthermore, as was discussed earlier, for $i \leq b-a$ the degrees of all line bundles in (3.92) are positive. Therefore, using eqs. (3.92), (3.25) and (3.26), for $i \leq b-a$ we have

$$
\begin{aligned}
& h^{0}\left(\mathcal{S}, \rho_{*} \mathcal{O}_{B}((a-2 i) \mathcal{S}+(b-3 i \mathcal{E}))\right)=\sum_{i=2}^{b-a} \sum_{j=b-a-i}^{b-3 i}(j+1)= \\
& \frac{1}{6}(b-a-1)\left(6-11 a+8 a^{2}+2 b-7 a b+2 b^{2}\right) .
\end{aligned}
$$

Now consider the case $i \geq b-a+1$. Then, it follows from eq. (3.92) that

$$
\rho_{*} \mathcal{O}_{B}((a-2 i) \mathcal{S}+(b-3 i \mathcal{E}))=\mathcal{L}_{+} \oplus \mathcal{L}_{-}
$$

where $\mathcal{L}_{+}$is the direct sum of the line bundle of non-negative degree

$$
\mathcal{L}_{+}=\mathcal{O}_{\mathcal{S}}(b-3 i) \oplus \cdots \oplus \mathcal{O}_{\mathcal{S}}(1) \oplus \mathcal{O}_{\mathcal{S}}
$$

and $\mathcal{L}_{-}$is the direct sum of line bundles of negative degree. From eqs. (3.25) and (3.26) we find

$$
\begin{aligned}
& h^{0}\left(\mathcal{S}, \rho_{*} \mathcal{O}_{B}((a-2 i) \mathcal{S}+(b-3 i) \mathcal{E})\right)=h^{0}\left(\mathcal{S}, \mathcal{L}_{+}\right)=\sum_{i=b-a+1}^{n} \sum_{j=0}^{b-3 i}(j+1)= \\
& \frac{1}{2}(n-b+a)\left(3 a^{2}+b^{2}-1-3 a b-3 a n+3 n^{2}\right) .
\end{aligned}
$$


Now combine the results of eqs. (3.99), (3.100) and (3.103) to obtain

$$
\begin{aligned}
& h^{0}\left(X, \mathcal{O}_{X}(\mathcal{C})\right)=\frac{n}{3}\left(4 n^{2}-1\right)+n a b-\left(n^{2}-2\right)(a+b)+a r\left(\frac{n^{2}}{2}-1\right)-\frac{n}{2} r a^{2}+ \\
& \frac{1}{6}(n-b+a)(n-b+a-1)(n-b+a+1) .
\end{aligned}
$$

Using eqs. (3.15), (3.98) and (3.104), we find that the number of moduli in the case

$$
r=1, \quad b<a+n-1
$$

is given by

$$
\begin{aligned}
& n(V)=\frac{n}{3}\left(4 n^{2}-1\right)+n a b-\left(n^{2}-2\right)(a+b)+\operatorname{ar}\left(\frac{n^{2}}{2}-1\right)-\frac{n}{2} r a^{2}-1+ \\
& \frac{1}{3}(n-b+a)(n-b+a-1)(n-b+a+1) .
\end{aligned}
$$

Note that, using eqs. (3.20) and (3.98), the last expression can be rewritten as

$$
h^{0}\left(X, \mathcal{O}_{X}(\mathcal{C})\right)=\chi_{E}\left(X, \mathcal{O}_{X}(\mathcal{C})\right)+h^{1}\left(X, \mathcal{O}_{X}(\mathcal{C})\right)
$$

This implies that both $h^{2}\left(X, \mathcal{O}_{X}(\mathcal{C})\right)$ and $h^{3}\left(X, \mathcal{O}_{X}(\mathcal{C})\right)$ must vanish. We will check our results by presenting an independent proof of this in Appendix A.. Before concluding this subsection, let us summarize the results. First, $\mathcal{C}$ being effective and irreducible requires that coefficients $a, b$ satisfy (3.70) for any choice of $n$ and $r$.

- If

$$
r=0,2 \quad \text { or } \quad r=1, b \geq a+n-1
$$

then

$$
\begin{gathered}
h^{0}\left(X, \mathcal{O}_{X}(\mathcal{C})\right)=\frac{n}{3}\left(4 n^{2}-1\right)+n a b-\left(n^{2}-2\right)(a+b)+a r\left(\frac{n^{2}}{2}-1\right)-\frac{n}{2} r a^{2}, \\
h^{1}\left(X, \mathcal{O}_{X}(\mathcal{C})\right)=0,
\end{gathered}
$$

and

$$
n(V)=\frac{n}{3}\left(4 n^{2}-1\right)+n a b-\left(n^{2}-2\right)(a+b)+\operatorname{ar}\left(\frac{n^{2}}{2}-1\right)-\frac{n}{2} r a^{2}-1 .
$$

- If

$$
r=1, \quad b<a+n-1
$$


then

$$
\begin{aligned}
& h^{0}\left(X, \mathcal{O}_{X}(\mathcal{C})\right)=\frac{n}{3}\left(4 n^{2}-1\right)+n a b-\left(n^{2}-2\right)(a+b)+a r\left(\frac{n^{2}}{2}-1\right)-\frac{n}{2} r a^{2}+ \\
& \frac{1}{6}(n-b+a)(n-b+a-1)(n-b+a+1) \\
& \quad h^{1}\left(X, \mathcal{O}_{X}(\mathcal{C})\right)=\frac{1}{6}(n-b+a)(n-b+a-1)(n-b+a+1)
\end{aligned}
$$

and

$$
\begin{aligned}
& n(V)=\frac{n}{3}\left(4 n^{2}-1\right)+n a b-\left(n^{2}-2\right)(a+b)+\operatorname{ar}\left(\frac{n^{2}}{2}-1\right)-\frac{n}{2} r a^{2}-1+ \\
& \frac{1}{3}(n-b+a)(n-b+a-1)(n-b+a+1) .
\end{aligned}
$$

Eqs. (3.111), (3.115) give a complete classification of the number of instanton moduli on Calabi-Yau manifolds elliptically fibered over the Hirzebruch surfaces $\mathbb{F}_{r}$ with $r=0,1,2$.

\subsection{Examples with Non-Positive Spectral Covers}

Let us give some examples of the above concepts. We will consider again the two GUT models with three generations presented as Example 1 and Example 2 in Section 2.

Example 1. Consider the vector bundle specified by $B=\mathbb{F}_{1}, G=S U(3)$, the spectral cover

$$
\mathcal{C} \in\left|3 \sigma+\pi^{*}(6 \mathcal{S}+10 \mathcal{E})\right|
$$

and

$$
\lambda=\frac{1}{2} .
$$

As discussed previously, coefficients $a=6, b=10$ do not satisfy condition (3.17) and, hence, spectral cover (3.116) is not positive. Using eqs. (3.113) and (3.114) we find that

$$
h^{0}\left(X, \mathcal{O}_{X}(\mathcal{C})\right)=70, \quad h^{1}\left(X, \mathcal{O}_{X}(\mathcal{C})\right)=0
$$

and, therefore,

$$
n(V)=69
$$

The number of generations and the five-brane class were computed in Example 1 in Section 2. 
Example 2. Consider the vector bundle specified by $B=\mathbb{F}_{1}, G=S U(3)$, the spectral cover

$$
\mathcal{C} \in\left|3 \sigma+\pi^{*}(8 \mathcal{S}+9 \mathcal{E})\right|
$$

and

$$
\lambda=\frac{3}{2} .
$$

As discussed previously, the coefficients $a=8, b=9$ do not satisfy (3.17) and, hence, spectral cover (3.120) is not positive. Using eqs. (3.113) and (3.114), we find that

$$
h^{0}\left(X, \mathcal{O}_{X}(\mathcal{C})\right)=64, \quad h^{1}\left(X, \mathcal{O}_{X}(\mathcal{C})\right)=1 .
$$

Therefore,

$$
n(V)=64 .
$$

The number of generations and the five-brane class were computed in Example 2 in Section 2 .

The number of moduli we obtain is smaller, roughly by a factor of two, than in the examples presented in [27]. Thus, by considering bundles with non-positive spectral cover, we significantly reduce the number of moduli.

\subsection{Bundles with the Minimal Number of Moduli}

Having found expressions for the number of instanton moduli which are valid for all spectral covers, it is natural to ask under what conditions $n(V)$ is minimal. First consider $h^{0}\left(X, \mathcal{O}_{X}(\mathcal{C})\right)$. Let $\mathcal{C}$ and $\mathcal{C}^{\prime}$ be two irreducible spectral covers. $\mathcal{C}$ being irreducible means that in the linear system $|\mathcal{C}|$ there are irreducible representatives. Also assume that

$$
\mathcal{C}^{\prime}=\mathcal{C}+\delta \mathcal{C}
$$

where $\delta \mathcal{C}$ is non-vanishing and effective. We want to understand the relation between $h^{0}\left(X, \mathcal{O}_{X}(\mathcal{C})\right)$ and $h^{0}\left(X, \mathcal{O}_{X}\left(\mathcal{C}^{\prime}\right)\right)$. The following fact is useful: holomorphic sections in $h^{0}\left(X, \mathcal{O}_{X}(\mathcal{C})\right)$ can be thought of as holomorphic sections in $h^{0}\left(X, \mathcal{O}_{X}\left(\mathcal{C}^{\prime}\right)\right)$ which vanish along $\delta \mathcal{C}$. In particular, this says that

$$
h^{0}\left(X, \mathcal{O}_{X}\left(\mathcal{C}^{\prime}\right)\right) \geq h^{0}\left(X, \mathcal{O}_{X}(\mathcal{C})\right),
$$

with equality holding if every section of $\mathcal{O}_{X}\left(\mathcal{C}^{\prime}\right)$ vanishes along $\delta \mathcal{C}$. Equality in (3.125) has a simple geometrical interpretation. It implies that the defining equation for every divisor 
in $\left|\mathcal{C}^{\prime}\right|$ factorizes, with one factor being the equation of $\delta \mathcal{C}$. It follows that all divisors in $\left|\mathcal{C}^{\prime}\right|$ are reducible. This contradicts our assumption that the spectral cover $\mathcal{C}^{\prime}$ is irreducible. Therefore

$$
h^{0}\left(X, \mathcal{O}_{X}\left(\mathcal{C}^{\prime}\right)\right)>h^{0}\left(X, \mathcal{O}_{X}(\mathcal{C})\right) .
$$

Recall from (3.70) that, for given $r$ and $n$, the minimal allowed values of the the coefficients $a$ and $b$ are

$$
a=2 n, \quad b=n(r+2) .
$$

Denote the associated "minimal" spectral cover by $\mathcal{C}$. It follows from (3.70) that any other allowed spectral cover is of the form (3.124) where $\delta \mathcal{C}$ is non-vanishing and effective. Therefore, (3.126) holds. Clearly, then, $h^{0}\left(X, \mathcal{O}_{X}(\mathcal{C})\right)$ is minimal when the coefficients $a$ and $b$ in the definition of the spectral cover (3.69) satisfy (3.127). It is important to note that neither coefficient $a$ nor $b$ in (3.127) satisfies the inequalities in (3.17). Hence, the minimal spectral cover is never positive. It is straightforward to check that for such values of $a$ and $b h^{1}\left(X, \mathcal{O}_{X}(\mathcal{C})\right)$ vanishes and, hence, it is also minimized. Furthermore, note that for $r=1$ the coefficients $a$ and $b$ satisfy the inequality $b \geq a+n-1$. Hence, for any $r$ and $n$, the number of moduli in the minimal case is of the form (3.111). Inserting (3.127) into (3.111) yields

$$
n_{\min }(V)=\frac{1}{3}\left(4 n^{3}+23 n-3\right) .
$$

It is easy to show that this expression is indeed an integer. Eq. (3.128) represents the minimal number of moduli for any rank $n$ vector bundle. Note that it is independent of $r$. Let us summarize our results.

- For

$$
r=0,1,2, \quad G=S U(n)
$$

and

$$
a=2 n, \quad b=n(r+2)
$$

the number of moduli is minimal and given by

$$
n_{\min }(V)=\frac{1}{3}\left(4 n^{3}+23 n-3\right) .
$$

Let us now consider an example. 


\subsection{Example with Minimal Number of Moduli}

Example 3. Consider the vector bundle specified by $B=\mathbb{F}_{r}, G=S U(3)$ and the spectral cover

$$
\mathcal{C} \in\left|3 \sigma+\pi^{*}(6 \mathcal{S}+3(r+2) \mathcal{E})\right|
$$

Then $n=3$ and the parameters $a$ and $b$ satisfy eq. (3.127). Therefore, we can use eq. (3.131) to evaluate the number of moduli. We find that

$$
n_{\min }(V)=58
$$

This is the minimal number of moduli for a rank 3 instanton on a Calabi-Yau threefold elliptically fibered over any Hirzebruch surface $\mathbb{F}_{r}, r=0,1,2$. Using eqs. (2.7), (2.18), (22.19) and (3.132), we find that the number of generations in this model is

$$
N_{\text {gen }}=0 .
$$

Furthermore, the five-brane class is

$$
[W]=\sigma \cdot \pi^{*}(18 \mathcal{S}+27 \mathcal{E})+96 F
$$

for every choice of parameter $\lambda$. Note that $[W]$ is effective.

Recall that the two examples in the previous subsection each had $n=3$ and $r=1$. Example 1 was specified by

$$
a=6, \quad b=10
$$

and had

$$
n(V)=69
$$

moduli. Similarly, Example 2 was specified by

$$
a=8, \quad b=9
$$

and had

$$
n(V)=64
$$

moduli. Since coefficients $b$ in (3.136) and $a$ in (3.138) do not satisfy (3.127), we expect $n(V)$ in each example to exceed 58 , which they do. 


\section{Small Instanton Transitions}

\subsection{Transition Moduli}

Consider a vacuum configuration of heterotic M-theory consisting of two end-of-the-world branes with vector bundles $V_{1}$ and $V_{2}$ and five-branes wrapped on the holomorphic curve

$$
[W]=\sigma \cdot \pi^{*} w+a_{f} F
$$

We assume that the vector bundle $V_{2}$ is trivial and $V_{1}$, which we denote by $V$, is defined by the spectral cover

$$
\mathcal{C} \in\left|n \sigma+\pi^{*} \eta\right|
$$

We will always take $\mathcal{C}$ to be effective and irreducible.

Consider any effective, irreducible curve component of $[W]$. It was shown in [26] that one can move this curve to a boundary brane and absorb it as a small instanton transition into the vector bundle $V$. This results in a new bundle $V^{\prime}$. A transition which absorbs only a horizontal component of $[W]$, that is, $\sigma \cdot \pi^{*} z$ where $z \subseteq w$, modifies the spectral cover (4.2) to

$$
\mathcal{C}^{\prime} \in\left|n \sigma+\pi^{*}(\eta+z)\right|
$$

which describes a new vector bundle $V^{\prime}$. Transitions of this type preserve the low-energy gauge group $H$, but change the third Chern class of the bundle and, hence, the number of families on the visible brane. Such transitions are called "chirality changing". It was also demonstrated in [26] these transitions do not alter the topological type of the line bundle $\mathcal{N}$. It is also possible to have a transition in which a purely vertical component of the five-brane curve gets absorbed [26]. However, in this case, the new bundle is reducible. The low-energy gauge group changes whereas the number of families does not. The number of moduli for these bundles was calculated in [29]. We will not consider such transitions in this paper.

An analogous, but simpler, transition takes place in type II string theories in the $D p-$ $D(p+4)$ system 61]. The $D p-D(p+4)$ system is describable by a supersymmetric field theory with eight supercharges. The moduli space of this system consists of two branches, the Coulomb branch and the Higgs branch. The Coulomb branch describes positions of the $D p$-brane away from the $D(p+4)$-brane. The Higgs branch describes how the $D p$-brane can get dissolved into the $D(p+4)$-brane. Geometrically, the Higgs branch is isomorphic to the one-instanton moduli space on a 4-manifold which is just the ADHM moduli space. In the heterotic M-theory case, the analog of the Coulomb branch is the moduli space of 
five-branes. The analog of the Higgs branch is the space of transition moduli. The concept of transition moduli was introduced in [27. They are the new moduli that arise after the transition and represent a Calabi-Yau threefold analog of the ADHM one-instanton moduli space. See 27] for various properties of the transition moduli.

The number of the transition moduli can be computed as

$$
n_{t m}=n\left(V^{\prime}\right)-n(V),
$$

where $n(V)$ is given by eq. (3.111) or eq. (3.115). Transition moduli appear to play an important role in heterotic compactifications. In [35, 36], the non-perturbative superpotentials for vector bundle moduli were studied. It was shown that the superpotential due to a string wrapped on an isolated curve $\sigma \cdot \pi^{*} \mathcal{S}$ in a Calabi-Yau manifold fibered over $\mathbb{F}_{r}$ is proportional to a polynomial of the transition moduli associated with the curve $\sigma \cdot \pi^{*} \mathcal{S}$. For example, in the case

$$
\begin{aligned}
& r=2, \quad n=3, \quad \lambda=\frac{3}{2}, \\
& \mathcal{C} \in\left|3 \sigma+\pi^{*}(a \mathcal{S}+b \mathcal{E})\right|
\end{aligned}
$$

with

$$
a>6, \quad b-2 a=2,
$$

the non-perturbative superpotential due to a string wrapped on the curve $\sigma \cdot \pi^{*} \mathcal{S}$ is given by

$$
W \propto \mathcal{R}^{4},
$$

where

$$
\mathcal{R}=\alpha_{1} \beta_{2} \gamma_{3}-\alpha_{1} \beta_{3} \gamma_{2}+\alpha_{2} \beta_{3} \gamma_{1}-\alpha_{2} \beta_{1} \gamma_{3}+\alpha_{3} \beta_{1} \gamma_{2}-\alpha_{3} \beta_{2} \gamma_{1}
$$

Here $\alpha_{i}, \beta_{j}, \gamma_{k}$ are nine transition moduli associated with the curve $\sigma \cdot \pi^{*} \mathcal{S}$. See [36] for details. However, all results and applications of transition moduli in [27, 35, 36] hold only for vector bundles with positive spectral cover. In the next subsection, we will define, and give a geometric meaning to, the transition moduli in the case of arbitrary, irreducible spectral covers.

\subsection{Geometric Interpretation of Transition Moduli for Arbitrary Irreducible Spectral Covers}

The number of instanton moduli was given in (3.15) by

$$
n(V)=\left(h^{0}\left(X, \mathcal{O}_{X}(\mathcal{C})\right)-1\right)+h^{1}\left(X, \mathcal{O}_{X}(\mathcal{C})\right),
$$


where $h^{0}\left(X, \mathcal{O}_{X}(\mathcal{C})\right)-1$ counts the parameters of the spectral cover $\mathcal{C}$ and $h^{1}\left(X, \mathcal{O}_{X}(\mathcal{C})\right)$ the moduli of the line bundle $\mathcal{N}$. The number of transition moduli is

$$
\begin{aligned}
& n_{t m}=n\left(V^{\prime}\right)-n(V)= \\
& {\left[h^{0}\left(X, \mathcal{O}_{X}\left(\mathcal{C}^{\prime}\right)\right)-h^{0}\left(X, \mathcal{O}_{X}(\mathcal{C})\right)\right]+\left[h^{1}\left(X, \mathcal{O}_{X}\left(\mathcal{C}^{\prime}\right)\right)-h^{1}\left(X, \mathcal{O}_{X}(\mathcal{C})\right)\right] .}
\end{aligned}
$$

In this subsection, we want to find a geometric interpretation for eq. (4.10). This can be important for calculating non-perturbative superpotentials, as was the case for positive spectral covers in refs. [35, 36]. First, consider the situation when

$$
h^{1}\left(X, \mathcal{O}_{X}\left(\mathcal{C}^{\prime}\right)\right)=h^{1}\left(X, \mathcal{O}_{X}(\mathcal{C})\right)=0
$$

Then

$$
n_{t m}=h^{0}\left(X, \mathcal{O}_{X}\left(\mathcal{C}^{\prime}\right)\right)-h^{0}\left(X, \mathcal{O}_{X}(\mathcal{C})\right)
$$

which coincides with the number of transition moduli of bundles with positive spectral cover [27]. Hence, this difference has the same interpretation as in [27], namely, that the difference in (4.12) is related to the moduli of the spectral cover restricted to the lift of the transition curve. More precisely, if the linear systems $\left|\mathcal{C}^{\prime}\right|$ and $|\mathcal{C}|$ are related as

$$
\left|\mathcal{C}^{\prime}\right|=\left|\mathcal{C}+\pi^{*} z\right|
$$

for some effective curve $z$, then [27]

$$
n_{t m}=h^{0}\left(\pi^{*} z,\left.\mathcal{O}_{X}\left(\mathcal{C}^{\prime}\right)\right|_{\pi^{*} z}\right) .
$$

To prove this, consider the short exact sequence

$$
0 \rightarrow \mathcal{O}_{X}(\mathcal{C}) \rightarrow \mathcal{O}_{X}\left(\mathcal{C}^{\prime}\right) \rightarrow \mathcal{O}_{\pi^{*} z}\left(\left.\mathcal{C}^{\prime}\right|_{\pi^{*} z}\right) \rightarrow 0
$$

The associated cohomology sequence is

$$
\begin{aligned}
& 0 \rightarrow H^{0}\left(X, \mathcal{O}_{X}(\mathcal{C})\right) \rightarrow H^{0}\left(X, \mathcal{O}_{X}\left(\mathcal{C}^{\prime}\right)\right) \rightarrow H^{0}\left(\pi^{*} z, \mathcal{O}_{\pi^{*} z}\left(\left.\mathcal{C}^{\prime}\right|_{\pi^{*} z}\right)\right) \\
& \stackrel{M}{\rightarrow} H^{1}\left(X, \mathcal{O}_{X}(\mathcal{C})\right) \stackrel{\alpha}{\rightarrow} H^{1}\left(X, \mathcal{O}_{X}\left(\mathcal{C}^{\prime}\right)\right) \rightarrow H^{1}\left(\pi^{*} z, \mathcal{O}_{\pi^{*} z}\left(\left.\mathcal{C}^{\prime}\right|_{\pi^{*} z}\right)\right) \rightarrow \ldots
\end{aligned}
$$

When eq. (4.11) holds, sequence (4.16) simplifies to

$$
0 \rightarrow H^{0}\left(X, \mathcal{O}_{X}(\mathcal{C})\right) \rightarrow H^{0}\left(X, \mathcal{O}_{X}\left(\mathcal{C}^{\prime}\right)\right) \rightarrow H^{0}\left(\pi^{*} z, \mathcal{O}_{\pi^{*} z}\left(\left.\mathcal{C}^{\prime}\right|_{\pi^{*} z}\right)\right) \rightarrow 0
$$

and it follows immediately that

$$
h^{0}\left(X, \mathcal{O}_{X}\left(\mathcal{C}^{\prime}\right)\right)-h^{0}\left(X, \mathcal{O}_{X}(\mathcal{C})\right)=h^{0}\left(\pi^{*} z,\left.\mathcal{O}_{X}\left(\mathcal{C}^{\prime}\right)\right|_{\pi^{*}}\right) .
$$


This proves eq. (4.14). It particular, it follows from (4.18) that

$$
\mathbb{P} H^{0}\left(X, \mathcal{O}_{X}(C)\right) \subset \mathbb{P} H^{0}\left(X, \mathcal{O}_{X}\left(C^{\prime}\right)\right) .
$$

That is, we can explicitly identify the moduli space of the vector bundle $V$ with a subspace of the moduli of bundle $V^{\prime}$. The normal directions to this subspace are simply given by global sections in $\mathcal{O}_{\pi^{*} z}\left(\left.\mathcal{C}^{\prime}\right|_{\pi^{*} z}\right)$.

Let us consider the general case when eq. (4.11) is no longer valid. The interpretation of transition moduli is now much more subtle. Again consider sequence (4.16) and the map $\alpha$. First, recall from (3.13) that

$$
H^{1}\left(\mathcal{C}, \mathcal{O}_{\mathcal{C}}\right)=H^{1}\left(X, \mathcal{O}_{X}(\mathcal{C})\right)^{*}
$$

An analogous condition holds if we replace $\mathcal{C}$ with $\mathcal{C}^{\prime}$. The map $\alpha$ in eq. (4.16) is

$$
\alpha: H^{1}\left(X, \mathcal{O}_{X}(\mathcal{C})\right) \rightarrow H^{1}\left(X, \mathcal{O}_{X}\left(\mathcal{C}^{\prime}\right)\right) .
$$

Dualizing $\alpha$ and using eq. (4.20), we get

$$
\alpha^{*}: H^{1}\left(\mathcal{C}^{\prime}, \mathcal{O}_{\mathcal{C}^{\prime}}\right) \rightarrow H^{1}\left(\mathcal{C}, \mathcal{O}_{\mathcal{C}}\right)
$$

Now $H^{1}\left(\mathcal{C}, \mathcal{O}_{\mathcal{C}}\right)$, up to a discrete lattice, is the moduli space of line bundles $\mathcal{N}$ on $\mathcal{C}$. Therefore, for fixed $\mathcal{C}$ and $\mathcal{C}^{\prime}, \alpha$ gives an explicit map between line bundles on $\mathcal{C}$ and $\mathcal{C}^{\prime}$. If we consider for a moment spectral covers $\mathcal{C}^{\prime}$ which are in the image of the inclusion (4.19), than $\mathcal{C}$ is contained in $\mathcal{C}^{\prime}$ and it is natural to interpret the map $\alpha^{*}$ as the restriction map. That is, it takes a line bundle on $\mathcal{C}^{\prime}$ and restricts it to $\mathcal{C}$.

At this point, it is useful to recall some linear algebra from Appendix B. Let $f$ be a linear map between two linear spaces $\mathcal{A}$ and $\mathcal{B}$. If we denote by $f^{*}$ the dual map, it follows from Appendix B that

$$
\begin{aligned}
& \operatorname{dim} \text { ker } f^{*}=\operatorname{dim} \text { coker } f, \\
& \operatorname{dim} \text { ker } f=\operatorname{dim} \text { coker } f^{*} .
\end{aligned}
$$

Let us use this fact to interpret the transition moduli. Sequence (4.16) can be split as follows

$$
0 \rightarrow H^{0}\left(X, \mathcal{O}_{X}(\mathcal{C})\right) \rightarrow H^{0}\left(X, \mathcal{O}_{X}\left(\mathcal{C}^{\prime}\right)\right) \rightarrow \operatorname{ker} M \rightarrow 0
$$

and

$$
0 \rightarrow i m M \rightarrow H^{1}\left(X, \mathcal{O}_{X}(\mathcal{C})\right) \stackrel{\alpha}{\rightarrow} H^{1}\left(X, \mathcal{O}_{X}\left(\mathcal{C}^{\prime}\right)\right) \rightarrow \text { coker } \alpha \rightarrow 0 .
$$


The first of these sequences states that $\operatorname{ker} M$ are the moduli of the spectral cover $\mathcal{C}^{\prime}$ which are not available for $\mathcal{C}$. That is, they are the new moduli that arise after the transition and modify the spectral cover. In other words, $\operatorname{dim} \operatorname{ker} M$ simply counts how many extra moduli the spectral cover $\mathcal{C}^{\prime}$ has comparing to $\mathcal{C}$. Obviously,

$$
\operatorname{dim} \text { ker } M=h^{0}\left(X, \mathcal{O}_{X}\left(\mathcal{C}^{\prime}\right)\right)-h^{0}\left(X, \mathcal{O}_{X}(\mathcal{C})\right) \text {. }
$$

We also need to understand $i m M$. Since the sequence (4.16) is exact, it follows that

$$
\text { im } M=\operatorname{ker} \alpha .
$$

Furthermore, from eqs. (4.23) we get

$$
\text { im } M=\text { coker } \alpha^{*} \text {. }
$$

As discussed previously, the map $\alpha^{*}$ relates line bundles on $\mathcal{C}^{\prime}$ to $\mathcal{C}$. Then coker $\alpha^{*}$, by definition, are those line bundle on $\mathcal{C}$ which cannot be related to line bundles on $\mathcal{C}^{\prime}$. The last space in (4.25) to interpret is coker $\alpha$. Since, by eq. (4.23),

$$
\text { coker } \alpha=\operatorname{ker} \alpha^{*} \text {, }
$$

coker $\alpha$ are just those non-trivial line bundles on $\mathcal{C}^{\prime}$ which correspond to trivial line bundles on $\mathcal{C}$. By definition, transition moduli are new moduli that arise after the small instanton transition. Therefore, to find the number of transition moduli we have to add the parameters of the spectral cover available for $\mathcal{C}^{\prime}$ but not for $\mathcal{C}$ (that is, $\operatorname{ker} M$ ) to the parameters of those line bundles which are non-trivial on $\mathcal{C}^{\prime}$ but trivial on $\pi^{*} z$ (that is, coker $\alpha$ ). However, there is a caveat. As established, $i m M$ is the set of those line bundles which exist on $\mathcal{C}$ but cannot be related to line bundles from $\mathcal{C}^{\prime}$. If im $M$ is non-zero, such moduli will disappear after the transition. Therefore, the number of transition moduli must be equal to

$$
n_{t m}=\operatorname{dim} \operatorname{ker} M+\operatorname{dim} \text { coker } \alpha-\operatorname{dim} \operatorname{im} M \text {. }
$$

This equation counts how many new moduli appear after the small instanton transition. Let us see whether eq (4.30) is equivalent to eq. (4.10). From the sequences (4.24) and (4.25), it follows that

$$
\operatorname{dim} \operatorname{ker} M=h^{0}\left(X, \mathcal{O}_{X}\left(\mathcal{C}^{\prime}\right)\right)-h^{0}\left(X, \mathcal{O}_{X}(\mathcal{C})\right)
$$

and

$$
\text { coker } \alpha-i m M=h^{1}\left(X, \mathcal{O}_{X}\left(\mathcal{C}^{\prime}\right)\right)-h^{1}\left(X, \mathcal{O}_{X}(\mathcal{C})\right) \text {. }
$$


Substituting eqs. (4.31) and (4.32) into eq. (4.30), we obtain precisely eq. (4.10).

Let us now summarize our interpretation of transition moduli for arbitrary spectral covers.

- Some transition moduli arise as new parameters of the spectral cover. Note that, in general, they do not correspond to the parameters of the spectral cover restricted to the lift of the curve $H^{0}\left(\pi^{*} z, \mathcal{O}_{\pi^{*} z}\left(\left.\mathcal{C}^{\prime}\right|_{\pi^{*}}\right)\right)$. They do so only if the map $\alpha$ is an isomorphism, as follows from the sequence (4.16). In this case, the map $M$ is the zero map and its kernel is the whole space $H^{0}\left(\pi^{*} z, \mathcal{O}_{\pi^{*} z}\left(\left.\mathcal{C}^{\prime}\right|_{\pi^{*} z}\right)\right)$. In particular, this happens when the spectral covers $\mathcal{C}^{\prime}$ and $\mathcal{C}$ are both positive.

- Some transition moduli arise as parameters of the line bundle $\mathcal{N}$ on $\mathcal{C}^{\prime}$ which correspond to the trivial line bundle on $\mathcal{C}$.

- Some moduli can disappear in the process of the transition. They correspond to moduli of those line bundles on $\mathcal{C}$ which are not related to line bundles on $\mathcal{C}^{\prime}$.

Two further remarks are in order. First, we note that during a small instanton transition the number of parameters of the spectral cover must always increase. Recall from Subsection 3.5. that the spectral covers before and after the transition, $\mathcal{C}$ and $\mathcal{C}^{\prime}$ respectively, are related by

$$
\mathcal{C}^{\prime}=\mathcal{C}+\delta \mathcal{C}
$$

where $\mathcal{C}, \mathcal{C}^{\prime}$ and $\delta \mathcal{C}$ are all effective and irreducible and, hence, that

$$
h^{0}\left(X, \mathcal{O}_{X}\left(\mathcal{C}^{\prime}\right)\right)>h^{0}\left(X, \mathcal{O}_{X}(\mathcal{C})\right)
$$

Therefore, the number of parameters of the spectral cover always becomes larger. Second, we note that this is not true for line bundle moduli. In the next subsection, we will give explicit examples of small instanton transition where the number of line bundle moduli decreases. This indicates that the moduli described in the last item above do indeed exist and, generically, that $i m$ does not vanish.

\subsection{Examples}

In this concluding subsection, we give several examples of chirality changing small instanton transitions involving various vacua with three generations. We will observe that the number of generations changes after the transition. 
Example A. In this example, we choose $B=\mathbb{F}_{1}, G=S U(3), a=6, b=9, \lambda=\frac{1}{2}$. Note that this case corresponds to Example 3 in Section 3 with $r=1$. From eqs. (2.7), (2.18), (2.19) we get

$$
N_{g e n}=0
$$

and

$$
[W]=\sigma \cdot \pi^{*}(18 \mathcal{S}+27 \mathcal{E})+96 F .
$$

From eqs. (3.109), (3.110) and (3.111) we obtain

$$
h^{0}\left(X, \mathcal{O}_{X}(\mathcal{C})\right)=59, \quad h^{1}\left(X, \mathcal{O}_{X}(\mathcal{C})\right)=0
$$

and

$$
n(V)=58 .
$$

A1. Let us make a transition with the curve $\sigma \cdot \pi^{*} \mathcal{E}$. The remaining five-brane class is

$$
\left[W^{\prime}\right]=\sigma \cdot \pi^{*}(18 \mathcal{S}+26 \mathcal{E})+96 F
$$

while the new spectral cover is given by

$$
a^{\prime}=6, \quad b^{\prime}=10 .
$$

This corresponds to Example 1 in Section 2. From eqs. (3.109), (3.110) and (3.111) we obtain

$$
h^{0}\left(X, \mathcal{O}_{X}\left(\mathcal{C}^{\prime}\right)\right)=70, \quad h^{1}\left(X, \mathcal{O}_{X}\left(\mathcal{C}^{\prime}\right)\right)=0
$$

and

$$
n(V)=69 .
$$

The number of transition moduli can be calculated by using eq. (4.42)

$$
n_{t m}=n\left(V^{\prime}\right)-n(V)=11 .
$$

The number of generations was computed in (2.27) and is given by

$$
N_{\text {gen }}=3 .
$$

This is an example of a pure spectral cover transition. It changes the number of generations from zero to three. 
A2. Now consider a transition with the curve $\sigma \cdot \pi^{*} \mathcal{S}$. The resulting five-brane class is

$$
\left[W^{\prime \prime}\right]=\sigma \cdot \pi^{*}(17 \mathcal{S}+27 \mathcal{E})+96 F
$$

and the new spectral cover is characterized by

$$
a^{\prime \prime}=7, \quad b^{\prime \prime}=9 .
$$

From eqs. (3.109), (3.110) and (3.111) we obtain

$$
h^{0}\left(X, \mathcal{O}_{X}\left(\mathcal{C}^{\prime \prime}\right)\right)=63, \quad h^{0}\left(X, \mathcal{O}_{X}\left(\mathcal{C}^{\prime \prime}\right)\right)=0
$$

and

$$
n(V)=62 .
$$

The number of transition moduli can be calculated by using eq. (4.42)

$$
n_{t m}=n\left(V^{\prime \prime}\right)-n(V)=4
$$

From eq. (2.19) we find that

$$
N_{\text {gen }}=1 .
$$

This is also an example of a pure spectral cover transition. It changes the number of generations from zero to one.

Example B. In this example, we choose $B=\mathbb{F}_{1}, G=S U(3), a=8, b=9, \lambda=\frac{3}{2}$. This corresponds to Example 2 in Section 2, where the number of generations and the five-brane class were computed and found to be

$$
N_{\text {gen }}=3
$$

and

$$
[W]=\sigma \cdot \pi^{*}(16 \mathcal{S}+27 \mathcal{E})+90 F .
$$

From eqs. (3.113), (3.114) and (3.115) we obtain

$$
h^{0}\left(X, \mathcal{O}_{X}(\mathcal{C})\right)=64, \quad h^{1}\left(X, \mathcal{O}_{X}(\mathcal{C})\right)=1
$$

and

$$
n(V)=64 .
$$

B1. Let us make a transition with the curve $\sigma \cdot \pi^{*} \mathcal{E}$. The resulting five-brane class is

$$
\left[W^{\prime}\right]=\sigma \cdot \pi^{*}(16 \mathcal{S}+26 \mathcal{E})+90 F .
$$


The new spectral cover is characterized by

$$
a^{\prime}=8, \quad b^{\prime}=10 .
$$

From eqs. (3.109), (3.110) and (3.111) we obtain

$$
h^{0}\left(X, \mathcal{O}_{X}\left(\mathcal{C}^{\prime}\right)\right)=81, \quad h^{1}\left(X, \mathcal{O}_{X}\left(\mathcal{C}^{\prime}\right)\right)=0
$$

and

$$
n(V)=80 \text {. }
$$

The number of transition moduli can be calculated by using eq. (4.42)

$$
n_{t m}=n\left(V^{\prime}\right)-n(V)=16 .
$$

From eq. (2.19), we find that the number of generations is

$$
N_{g e n}=18
$$

Note that, as always, the number of spectral cover moduli has increased. However, this is a clear example of a transition where the number of line bundle moduli decreases.

B2. Now consider a transition with the curve $\sigma \cdot \pi^{*} \mathcal{S}$. The resulting five-brane class is

$$
\left[W^{\prime \prime}\right]=\sigma \cdot \pi^{*}(15 \mathcal{S}+27 \mathcal{E})+90 F
$$

and the new spectral cover is characterized by

$$
a^{\prime \prime}=9, \quad b^{\prime \prime}=9 .
$$

From eqs. (3.113), (3.114) and (3.115) we obtain

$$
h^{0}\left(X, \mathcal{O}_{X}\left(\mathcal{C}^{\prime \prime}\right)\right)=66, \quad h^{1}\left(X, \mathcal{O}_{X}\left(\mathcal{C}^{\prime \prime}\right)\right)=4
$$

and

$$
n(V)=69 .
$$

The number of transition moduli can be calculated by using eq. (4.42)

$$
n_{t m}=n\left(V^{\prime \prime}\right)-n(V)=5 .
$$

From eq. (2.19) we find that

$$
N_{g e n}=0 .
$$

Note that, in this example, the number of both the spectral cover and the line bundles parameters increases. 


\section{Conclusion}

In this paper, we have given a complete classification of the number of instanton moduli for Calabi-Yau threefolds elliptically fibered over the Hirzebruch surfaces $\mathbb{F}_{r}, r=0,1,2$. The

results are presented in eqs. (3.111) and (3.115). An expression for the minimal possible number of moduli for a vector bundle of fixed $r$ and $n$ was presented. It is independent of $r$ and given by

$$
n_{\min }(V)=\frac{1}{3}\left(4 n^{3}+23 n-3\right) .
$$

For example, for $n=3$ the minimal number of vector bundle moduli is 58 . Hence, in GUT theories built on such vacua, the number of instanton moduli is always rather large. It seems unlikely that this number can substantially be reduced by considering instantons on CalabiYau threefolds fibered over different bases. However, it is natural to expect that the number of moduli will be greatly reduced for standard model-like bundles on Calabi-Yau threefolds with non-trivial discrete homotopy group [12, 13, 14, 15, 16, 17, 18. Such bundles are more rigid and one does not expect many moduli. We also gave an interpretation for transition moduli. Such moduli arise after the small instanton transition and represent a Calabi-Yau threefold generalization of the ADHM one-instanton moduli space. We saw that the number of transition moduli in certain cases can be reduced comparing to previous results in [27] and can be as few as four. We supplemented all of these concepts with concrete examples.

\section{Appendix A: Consistency Checks}

In this Appendix, we present some consistency checks of eqs. (3.111) and (3.115). First, note that the positivity conditions (3.17) are a special case of the conditions stated in eq. (3.108). Therefore, eq. (3.111) should be valid for positive spectral covers. This is indeed correct as eq. (3.111) coincides with eq. (3.21). The second consistency check we can perform is the following. Comparing eqs. (3.111) and (3.115) with the expression for the Euler characteristic (3.20), we see that eq. (3.111) can be written as

$$
n(V)=\chi_{E}\left(X, \mathcal{O}_{X}(\mathcal{C})\right)-1
$$

whereas eq. (3.115) can be expressed as

$$
n(V)=\chi_{E}\left(X, \mathcal{O}_{X}(\mathcal{C})\right)-1+2 h^{1}\left(\left(X, \mathcal{O}_{X}(\mathcal{C})\right)\right.
$$

This indicates that

$$
H^{2}\left(X, \mathcal{O}_{X}(\mathcal{C})\right)=H^{3}\left(X, \mathcal{O}_{X}(\mathcal{C})\right)=0
$$


for all spectral covers (of course, we always assume that conditions (2.10)- (2.12) are satisfied) as mentioned in eqs. (3.83) and (3.107) above . Let us prove eq. (6.3) directly, using independent methods. Start with $H^{2}\left(X, \mathcal{O}_{X}(\mathcal{C})\right)$. From Leray spectral sequences, it follows that

$$
H^{2}\left(X, \mathcal{O}_{X}(\mathcal{C})\right)=H^{2}\left(B, \pi_{*} \mathcal{O}_{X}(\mathcal{C})\right)
$$

We have used the facts that

$$
H^{0}\left(B, R^{2} \pi_{*} \mathcal{O}_{X}(\mathcal{C})\right)=0
$$

since the fiber of the projection $\pi$ is one-dimensional and

$$
H^{1}\left(B, R^{1} \pi_{*} \mathcal{O}_{X}(\mathcal{C})\right)=0
$$

as was proven previously in eq (3.44). Next, we relate $H^{2}\left(X, \mathcal{O}_{X}(\mathcal{C})\right)$ to $H^{2}\left(\mathcal{S}, \rho_{*} \pi_{*} \mathcal{O}_{X}(\mathcal{C})\right)$. Note that

$$
H^{0}\left(\mathcal{S}, R^{2} \rho_{*} \pi_{*} \mathcal{O}_{X}(\mathcal{C})\right)=0
$$

since the fiber of the projection $\rho$, the curve $\mathcal{E}$, is one-dimensional and

$$
H^{1}\left(\mathcal{S}, R^{1} \rho_{*} \pi_{*} \mathcal{O}_{X}(\mathcal{C})\right)=0
$$

as shown previously (see eq. (3.53) and discussion just above it). Using this and a Leray spectral sequence, we find that

$$
H^{2}\left(B, \pi_{*} \mathcal{O}_{X}(\mathcal{C})\right)=H^{2}\left(\mathcal{S}, \rho_{*} \pi_{*} \mathcal{O}_{X}(\mathcal{C})\right)
$$

This vanishes since $\mathcal{S}$ is one-dimensional. Combining this result with eq. (6.4) proves that

$$
H^{2}\left(X, \mathcal{O}_{X}(\mathcal{C})\right)=0
$$

Similarly, we can prove that $H^{3}\left(X, \mathcal{O}_{X}(\mathcal{C})\right)$ vanishes. To see this, note that

$$
H^{2}\left(B, R^{1} \pi_{*} \mathcal{O}_{X}(\mathcal{C})\right)=0
$$

since the sheaf $R^{1} \pi_{*} \mathcal{O}(\mathcal{C})$ vanishes (see eq. (3.44)) and

$$
H^{1}\left(B, R^{2} \pi_{*} \mathcal{O}_{X}(\mathcal{C})\right)=H^{0}\left(B, R^{3} \pi_{*} \mathcal{O}_{X}(\mathcal{C})\right)=0
$$

since the fiber of $\pi$ is one-dimensional. Leray spectral sequences then imply that

$$
H^{3}\left(X, \mathcal{O}_{X}(\mathcal{C})\right)=H^{3}\left(B, \pi_{*} \mathcal{O}_{X}(\mathcal{C})\right)
$$


But

$$
H^{3}\left(B, \pi_{*} \mathcal{O}_{X}(\mathcal{C})\right)=0
$$

since $B$ is a surface. This proves that

$$
H^{3}\left(X, \mathcal{O}_{X}(\mathcal{C})\right)=0 .
$$

Hence, eqs. (3.111) and (3.115) are indeed consistent with eqs. (6.1) and (6.2).

\section{Appendix B: Some Linear Algebra}

Let $f$ be a map between two linear spaces $\mathcal{A}$ and $\mathcal{B}$. Then, it is known that there exists the following short exact sequence

$$
0 \rightarrow \operatorname{ker} f \rightarrow \mathcal{A} \stackrel{f}{\rightarrow} \mathcal{B} \rightarrow \text { coker } f \rightarrow 0 .
$$

Dualizing this sequence, we get

$$
0 \rightarrow(\operatorname{coker} f)^{*} \rightarrow \mathcal{B}^{*} \stackrel{f^{*}}{\rightarrow} \mathcal{A}^{*} \rightarrow(\text { ker } f)^{*} \rightarrow 0
$$

On the other hand, replacing $f$ by $f^{*}, \mathcal{A}$ by $\mathcal{B}^{*}$ and $\mathcal{B}$ by $\mathcal{A}^{*}$ in (7.1) we obtain

$$
0 \rightarrow \operatorname{ker} f^{*} \rightarrow \mathcal{B}^{*} \stackrel{f^{*}}{\rightarrow} \mathcal{A}^{*} \rightarrow \text { coker } f^{*} \rightarrow 0 .
$$

Comparing eqs. (17.2) and (7.3) we conclude that

$$
\begin{aligned}
& \operatorname{dim} \text { ker } f^{*}=\operatorname{dim} \text { coker } f, \\
& \operatorname{dim} \text { ker } f=\operatorname{dim} \text { coker } f^{*} .
\end{aligned}
$$

\section{Acknowledgment}

The work of E. I. B. is supported by NSF grant PHY-0070928. The work of B. A. O. is supported in part by the Department of Physics and the Math/Physics Research group at the University of Pennsylvania under cooperative research agreement No. DE-FG02-95ER40893 with the U. S. Department of Energy and an NSF Focused Research Grant DMS0139799 for "The Geometry of Superstrings". The work of R. R. is supported by DMS-0244464. 


\section{References}

[1] E. Witten, Strong Coupling Expansion Of Calabi-Yau Compactification, Nucl.Phys. B471 (1996) 135-158 hep-th/9602070.

[2] A. Lukas, B. A. Ovrut and D. Waldram, On the Four-Dimensional Effective Action of Strongly Coupled Heterotic String Theory, Nucl.Phys. B532 (1998) 43-82 hep-th/9710208.

[3] A. Lukas, B. A. Ovrut, K.S. Stelle and D. Waldram, The Universe as a Domain Wall, Phys.Rev. D59 (1999) 086001 hep-th/9803235.

[4] A. Lukas, B. A. Ovrut, K. S. Stelle and D. Waldram, Heterotic M-theory in Five Dimensions, Nucl.Phys. B552 (1999) 246-290 hep-th/9806051.

[5] A. Lukas, B. A. Ovrut and D. Waldram, Non-standard embedding and five-branes in heterotic M-Theory, Phys.Rev. D59 (1999) 106005 hep-th/9808101.

[6] P. Horava and E. Witten, Heterotic and Type I String Dynamics from Eleven Dimensions, Nucl.Phys. B460 (1996) 506-524 hep-th/9510209.

[7] P. Horava and E. Witten, Eleven-Dimensional Supergravity on a Manifold with Boundary, Nucl.Phys. B475 (1996) 94-114 hep-th/9603142.

[8] R. Friedman, J. Morgan and E. Witten, Vector Bundles And F Theory, Commun.Math.Phys. 187 (1997) 679-743 hep-th/9701162.

[9] R. Donagi, Principal bundles on elliptic fibrations, Asian J. Math. Vol. 1 (June 1997), 214-223 alg-geom/9702002.

[10] R. Donagi, A. Lukas, B. A. Ovrut and D. Waldram, Non-Perturbative Vacua and Particle Physics in M-Theory, JHEP 9905 (1999) 018 hep-th/9811168.

[11] R. Donagi, A. Lukas, B. A. Ovrut and D. Waldram, Holomorphic Vector Bundles and Non-Perturbative Vacua in M-Theory, JHEP 9906 (1999) 034 hep-th/9901009.

[12] R. Donagi, B. A. Ovrut, T. Pantev and D. Waldram, Standard-Model Bundles on NonSimply Connected Calabi-Yau Threefolds, JHEP 0108 (2001) 053 hep-th/0008008.

[13] R. Donagi, B. A. Ovrut, T. Pantev and D. Waldram, Standard-model bundles, Adv.Theor.Math.Phys. 5 (2002) 563-615 math.AG/0008010. 
[14] R. Donagi, B. A. Ovrut, T. Pantev and D. Waldram, Spectral involutions on rational elliptic surfaces, Adv.Theor.Math.Phys. 5 (2002) 499-561 math.AG/0008011.

[15] B. A. Ovrut, T. Pantev and R. Reinbacher, Torus-Fibered Calabi-Yau Threefolds with Non-Trivial Fundamental Group, JHEP 0305 (2003) 040 hep-th/0212221.

[16] B. A. Ovrut, T. Pantev and R. Reinbacher, Invariant Homology on Standard Model Manifolds, JHEP 0401 (2004) 059 hep-th/0303020.

[17] R. Donagi, B. A. Ovrut, T. Pantev and R. Reinbacher, SU(4) Instantons on Calabi-Yau Threefolds with $Z_{2} x Z_{2}$ Fundamental Group, JHEP 0401 (2004) 022 hep-th/0307273.

[18] V. Braun, B. A. Ovrut, T. Pantev and R. Reinbacher, Elliptic Calabi-Yau Threefolds with $Z_{3} x Z_{3}$ Wilson Lines hep-th/0410055.

[19] A. Lukas, B. A. Ovrut and D. Waldram, Non-standard embedding and five-branes in heterotic M-Theory, Phys.Rev. D59 (1999) 106005 hep-th/9808101.

[20] R. Donagi, B. A. Ovrut and D. Waldram, Moduli Spaces of Fivebranes on Elliptic Calabi-Yau Threefolds, JHEP 9911 (1999) 030 hep-th/9904054.

[21] Z. Lalak, S. Pokorski and S. Thomas, Beyond the Standard Embedding in M-Theory on $S^{1} / Z_{2}$, Nucl.Phys. B549 (1999) 63-97 hep-ph/9807503.

[22] J.-P. Derendinger and R. Sauser, A Five-brane Modulus in the Effective N=1 Supergravity of M-Theory, Nucl.Phys. B598 (2001) 87-114 hep-th/0009054.

[23] A. Grassi, Z. Guralnik and B. A. Ovrut, Knots, Braids and BPS States in M-Theory, JHEP 0206 (2002) 023 hep-th/0110036.

[24] N. Seiberg and E. Witten, Comments on String Dynamics in Six Dimensions, Nucl.Phys. B471 (1996) 121-134 hep-th/9603003.

[25] S. Kachru and E. Silverstein, Chirality Changing Phase Transitions in 4d String Vacua, Nucl.Phys. B504 (1997) 272-284 hep-th/9704185.

[26] B. A. Ovrut, T. Pantev and J. Park, Small Instanton Transitions in Heterotic M-Theory, JHEP 0005 (2000) 045 hep-th/0001133.

[27] E. I. Buchbinder, R. Donagi and B. A. Ovrut, Vector Bundle Moduli and Small Instanton Transitions, JHEP 0206 (2002) 054 hep-th/0202084. 
[28] B. Andreas and D. Hernandez Ruiperez, Comments on N=1 Heterotic String Vacua, Adv.Theor.Math.Phys. 7 (2004) 751-786 hep-th/0305123.

[29] Y.-H. He, B. A. Ovrut and R. Reinbacher, The Moduli of Reducible Vector Bundles, JHEP 0403 (2004) 043 hep-th/0306121.

[30] K. Becker, M. Becker and A. Strominger, Fivebranes, Membranes and Non-Perturbative String Theory, Nucl.Phys. B456 (1995) 130-152 hep-th/9507158.

[31] E. Witten, Non-Perturbative Superpotentials In String Theory, Nucl.Phys. B474 (1996) 343-360 hep-th/9604030.

[32] E. Lima, B. A. Ovrut, J. Park and R. Reinbacher, Non-Perturbative Superpotentials from Membrane Instantons in Heterotic M-Theory, Nucl.Phys. B614 (2001) 117-170 hep-th/0101049.

[33] E. Lima, B. Ovrut and J. Park, Five-Brane Superpotentials in Heterotic M-Theory, Nucl.Phys. B626 (2002) 113-164 hep-th/0102046.

[34] G. Moore, G. Peradze and N. Saulina, Instabilities in heterotic M-theory induced by open membrane instantons, Nucl.Phys. B670 (2003) 27-89 hep-th/0206092.

[35] E. I. Buchbinder, R. Donagi and B. A. Ovrut, Superpotentials for Vector Bundle Moduli, Nucl.Phys. B653 (2003) 400-420 hep-th/0205190.

[36] E. I. Buchbinder, R. Donagi and B. A. Ovrut, Vector Bundle Moduli Superpotentials in Heterotic Superstrings and M-Theory, JHEP 0207 (2002) 066 hep-th/0206203.

[37] C. Beasley and E. Witten, Residues and World-Sheet Instantons, JHEP 0310 (2003) 065 hep-th/0304115.

[38] P. Horava, Gluino Condensation in Strongly Coupled Heterotic String Theory, Phys.Rev. D54 (1996) 7561-7569 hep-th/9608019.

[39] A. Lukas, B. A. Ovrut and D. Waldram, Gaugino Condensation in $M$-theory on $S^{1} / Z_{2}$, Phys.Rev. D57 (1998) 7529-7538 hep-th/9711197.

[40] A. Lukas, B. A. Ovrut and D. Waldram, Five-Branes and Supersymmetry Breaking in M-Theory, JHEP 9904 (1999) 009 hep-th/9901017. 
[41] Z. Lalak and S. Thomas, Gaugino Condensation, Moduli Potentials and Supersymmetry Breaking in M-Theory Models, Nucl.Phys. B515 (1998) 55-72 hep-th/9707223.

[42] Z. Lalak and S. Thomas, Scales of Gaugino Condensation and Supersymmetry Breaking in Nonstandard M-Theory Embeddings, Nucl.Phys. B575 (2000) 151-176 hep-th/9908147.

[43] H. P. Nilles, Gaugino Condensation and SUSY Breakdown, Lectures at Cargese School of Physics and Cosmology, Cargese, France, August 2003 hep-th/0402022.

[44] G. Curio and A. Krause, G-Fluxes and Non-Perturbative Stabilisation of Heterotic MTheory, Nucl.Phys. B643 (2002) 131-156 hep-th/0108220.

[45] E. I. Buchbinder and B. A. Ovrut, Vacuum Stability in Heterotic M-Theory, Phys.Rev. D69 (2004) 086010 hep-th/0310112.

[46] S. Gukov, S. Kachru, X. Liu and L. McAllister, Heterotic Moduli Stabilization with Fractional Chern-Simons Invariants, Phys.Rev. D69 (2004) 086008 hep-th/0310159.

[47] M. Becker, G. Curio and A. Krause, De Sitter Vacua from Heterotic M-Theory, Nucl.Phys. B693 (2004) 223-260 hep-th/0403027.

[48] E. I. Buchbinder, Raising Anti de Sitter Vacua to de Sitter Vacua in Heterotic M-Theory, Phys. Rev. D 70, 066008 (2004) hep-th/0406101.

[49] R. Donagi, Y.-H. He, B. A. Ovrut and R. Reinbacher, Moduli Dependent Spectra of Heterotic Compactifications, Phys.Lett. B598 (2004) 279-284 hep-th/0403291.

[50] R. Donagi, Y.-H. He, B. A. Ovrut and R. Reinbacher, The Particle Spectrum of Heterotic Compactifications hep-th/0405014.

[51] R. Donagi, Y.-H. He, B. A. Ovrut and R. Reinbacher, Higgs Doublets, Split Multiplets and Heterotic $S U(3)_{C} x S U(2)_{L} x U(1)_{Y}$ Spectra hep-th/0409291.

[52] J. Khoury, B. A. Ovrut, P. J. Steinhardt and N. Turok, The Ekpyrotic Universe: Colliding Branes and the Origin of the Hot Big Bang, Phys.Rev. D64 (2001) 123522 hep-th/0103239.

[53] J. Khoury, B. A. Ovrut, N. Seiberg, P. J. Steinhardt and N. Turok, From Big Crunch to Big Bang, Phys.Rev. D65 (2002) 086007 hep-th/0108187. 
[54] J. Khoury, B. A. Ovrut, P. J. Steinhardt and N. Turok, Density Perturbations in the Ekpyrotic Scenario, Phys.Rev. D66 (2002) 046005 hep-th/0109050.

[55] D. R. Morrison and C. Vafa, Compactifications of F-Theory on Calabi-Yau Threefolds - II, Nucl.Phys. B476 (1996) 437-469 hep-th/9603161.

[56] S. Donaldson, Proc. London Math. Soc. 3 (1985) 1.

[57] K. Uhlenbeck and S.T. Yau, Comm. Pure App. Math. 39 (1986) 257; Comm. Pure App. Math. 42 (1986) 703.

[58] G. Curio, Chiral matter and transitions in heterotic string models, Phys.Lett. B435 (1998) 39-48 hep-th/9803224.

[59] B. Andreas, On Vector Bundles and Chiral Matter in N=1 Heterotic Compactifications, JHEP 9901 (1999) 011 hep-th/9802202.

[60] P. Griffiths and J. Harris, Principles of Algebraic Geometry, John Wiley and Sons, 1994.

[61] M. R. Douglas, Branes within Branes, in Cargese 1997, Strings, branes and dualities 267-275 hep-th/9512077. 